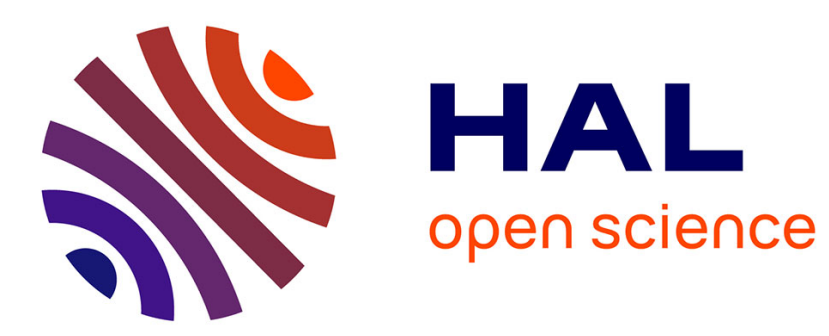

\title{
Exergy-Based Performance Assessment of the NASA Common Research Model
}

\author{
Aurélien Arntz, David Hue
}

\section{To cite this version:}

Aurélien Arntz, David Hue. Exergy-Based Performance Assessment of the NASA Common Research Model. AIAA Journal, 2016, 54 (1), pp.88-100. 10.2514/1.J054127 . hal-01917581

\section{HAL Id: hal-01917581 \\ https://hal.science/hal-01917581}

Submitted on 9 Nov 2018

HAL is a multi-disciplinary open access archive for the deposit and dissemination of scientific research documents, whether they are published or not. The documents may come from teaching and research institutions in France or abroad, or from public or private research centers.
L'archive ouverte pluridisciplinaire HAL, est destinée au dépôt et à la diffusion de documents scientifiques de niveau recherche, publiés ou non, émanant des établissements d'enseignement et de recherche français ou étrangers, des laboratoires publics ou privés. 


\title{
Exergy-Based Performance Assessment of the NASA Common Research Model
}

\author{
Aurélien Arntz* and David Hue \\ ONERA - The French Aerospace Lab, 92190 Meudon, France
}

DOI: $\underline{10.2514 / 1 . J 054127}$

\begin{abstract}
Aircraft have evolved into extremely complex systems that require adapted methodologies and tools for efficient design processes. A theoretical formulation based on exergy management has been recently proposed by Arntz et al. for assessing the aerothermopropulsive performance of future aircraft configurations. The present article focuses on the validation of its numerical implementation in a FORTRAN code for the postprocessing of Reynolds-averaged Navier-Stokes flow solutions. The flow around the wing-body NASA Common Research Model is assessed in terms of anergy destruction. A $2 \mathrm{MW}$ work potential associated with the lift-induced vortices is identified in the wake of the airplane. Subsequently, a six-level grid convergence study enables determining the robustness and accuracy of the exergy postprocessing code. The introduction and calibration of a numerical correction allows to account for the spurious numerical vortex dissipation and to obtain an accuracy similar to the traditional near-field drag method. Finally, the postprocessing code is validated for drag prediction against computational fluid dynamics and experimental wind-tunnel data.
\end{abstract}

\section{Nomenclature}

$\dot{\mathcal{A}}_{\text {tot }} \quad=$ rate of total anergy generation

$\dot{\mathcal{A}}_{w}=$ rate of anergy generation by shock waves

$\dot{\mathcal{A}}_{\nabla T}=$ rate of anergy generation by thermal mixing

$\dot{\mathcal{A}}_{\phi} \quad=$ rate of anergy generation by viscous dissipation

$b=$ semispan

$c=$ superscript for numerically corrected quantity

$C V=$ control volume

$D=$ aerodynamic drag

$d c=$ drag counts

$d_{T P}=$ transverse plane distance

$\dot{E}_{p}=$ boundary pressure-work rate

$\dot{E}_{u}=$

$\dot{E}_{v} \quad=$

$h_{i}=$

$k=$

n $=$

$p c=$

$s=$

V. $=$

$\Delta \dot{\mathcal{A}}=$

$\delta()=$

$\varepsilon \quad=$

$\dot{\mathcal{E}}_{m}=$

$\dot{\mathcal{E}}_{\text {th }}=$

$\overline{\bar{\tau}} \quad=$

$\Phi=$

Subscript

$\infty=$

$=$ quantity at freestream conditions

\author{
streamwise kinetic energy deposition rate \\ transverse kinetic energy deposition rate \\ mass specific total enthalpy \\ medium thermal conductivity \\ unit normal vector \\ power counts \\ mass specific entropy \\ fluid velocity vector [equal to $\left(V_{\infty}+u\right) \boldsymbol{x}, v \boldsymbol{y}, w \boldsymbol{z}$ ] \\ rate of missing anergy, not captured \\ quantity relative to freestream (equal to ()$\left.-()_{\infty}\right)$ \\ mass specific flow exergy (equal to $\delta h_{i}-T_{\infty} \delta s$ ) \\ rate of mechanical exergy outflow \\ rate of thermal exergy outflow \\ viscous stress tensor \\ dissipation rate per unit volume
}

Received 12 December 2014; revision received 6 May 2015; accepted for publication 7 May 2015; published online 24 July 2015. Copyright (C) 2015 by the authors. Published by the American Institute of Aeronautics and Astronautics, Inc., with permission. Copies of this paper may be made for personal or internal use, on condition that the copier pay the $\$ 10.00$ per-copy fee to the Copyright Clearance Center, Inc., 222 Rosewood Drive, Danvers, MA 01923; include the code 1533-385X/15 and \$10.00 in correspondence with the CCC.

*Ph.D. Student, Department of Applied Aerodynamics; aurelien.arntz@ gmail.com. Member AIAA.

${ }^{\dagger}$ Research Engineer, Department of Applied Aerodynamics; david.hue@ onera.fr. Member AIAA.

\section{Introduction}

A CENTURY after the first commercial flight made in January 1914, the air transportation industry has undergone fantastic growth, especially since the early 1950s from which time air traffic has doubled every 15-20 years. Most forecasts predict a continuing 5\% per annum growth, at least for the two coming decades. Such growth has raised environmental concern and driven international institutions to set ambitious objectives of performance. Among these objectives has emerged a dramatic reduction in both fuel combustion and noise/pollutant emissions. It is likely that the continuous improvement of the conventional tube-and-wings configuration will not be sufficient to satisfy these new constraints. One of the very promising configurations is probably the blended-wing body featuring a boundary-layer ingestion propulsion [1,2]. However, this configuration is rather unconventional, and current tools and methodologies become inapplicable to it. Especially, the conventional drag/thrust book keepings [3,4], used to separate the task of the airframe designer from the one of the propulsion engineers, becomes excessively ambiguous for such highly integrated propulsion systems [5-7]. This lack of suitable methodologies prevents an accurate estimation of the benefit given by a complete advanced configuration and represents the main motivation for deriving the present performance prediction formulation.

A new formulation has recently been proposed by Arntz et al. [8] to provide a global approach for the design of future configurations by taking into account the thermal energy in addition to the mechanical energy. The combination of the first and second laws of thermodynamic enables an exergy analysis that starts by splitting any form of energy in two parts: a first part (theoretically) fully convertible into mechanical work and a second part that is (theoretically) impossible to be converted into mechanical work. Following the work of Rant [9] to establish an international terminology, the words "exergy" and "anergy" have been adopted to represent these two parts. As exergy is the part of energy that has economic value [10], it is the only part worth managing carefully. The approach consists of considering that it is the usage and loss of thermodynamic work potential that drives virtually every aspect of a vehicle's environmental and economic performance [11]. Far from being an obscure scientific notion, the exergy analysis yields a fundamental definition of loss in an engineering perspective: exergy destruction, or equivalently anergy generation, by irreversible phenomena. In the literature, only a few aerospace configurations have been examined with Reynolds-averaged Navier-Stokes (RANS) flow computations from an exergy/anergy standpoint [12-16]. The authors also investigated unpowered airframe configurations in [17], and the concept of wake ingestion was analyzed in [18]. Other applications include the integration of a heat exchanger on aircraft [19]. 
The typical configuration for which the exergy balance has been proposed is a blended-wing body with a boundary-layer ingesting propulsion system like the one investigated by the authors in [20]. One required step toward the treatment of such unconventional architecture is to get confidence in the postprocessing code in which the formulation has been implemented. To identify its strength and weaknesses, the code should be tested on a well-documented application for which well-tried methods can be applied. The aerodynamic performance assessment of an unpowered configuration (airframe) has the advantage of allowing well-tried methods to which drag prediction can be compared. That geometry should be representative of an industryrelevant airplane. One other criterion to determine the sensitivity of the code to grid quality would be to allow for a grid refinement study. Finally, the ideal test case would have been assessed by other methods, both numerical and experimental. As a consequence, the present article deals with the validation of the postprocessing code on case 1 of the Fifth Drag Prediction Workshop [21].

First, two well-validated drag prediction methods are presented, and the theoretical formulation of the new method based on exergy management is introduced. Their numerical implementation in a postprocessing code is then discussed. The test case selected is presented in terms of geometry, flow regime, and numerics. Subsequently, a qualitative and quantitative flowfield analysis is made in terms of exergy outflows and anergy generation. A grid convergence study allows determining the sensitivity of the code to grid density, and a numerical correction for the viscous and thermal anergy production is introduced and calibrated. Finally, validation of the code is made with reference to the near-field drag method and with considerations on wind-tunnel experimental data.

\section{Review of Formulations and Numerical Implementation}

In the present application, the newly proposed exergy-based formulation is compared to two more traditional formulations based on momentum considerations which are reminded next. Then, the key aspects of the exergy-based formulation are introduced.

\section{A. Near-Field and Far-Field Drag Approaches}

The determination of the drag experienced by an unpowered configuration has historically been made by a force balance. There are two main families of drag prediction and breakdown: a near-field approach and a far-field approach.

The most straightforward method for evaluation the drag experienced by a body is by a direct integration of the stresses at its surface that yields a breakdown into pressure $\operatorname{drag} D_{p}$ and friction $\operatorname{drag} D_{f}$ :

$$
D_{\mathrm{nf}}:=D_{p}+D_{f}
$$

Numerically speaking, this approach is unambiguous and straightforward but highly dependent on mesh discretization, and therefore it is known to suffer from inaccuracy on coarse meshes.
There is a great variety of far-field drag methods [22] since the first suggestion of Betz [23] to manipulate the rate of change of momentum evaluated on a surface downstream of the body under analysis. As this surface is usually located within a few body lengths downstream of the geometry, or even less, the methods are actually not too far field. The one developed by Destarac [24] and van der Vooren and Destarac [25] will be used as a second reference for comparison. This method introduces thermodynamic relations in the momentum relation to obtain a decomposition into viscous drag $D_{v}$, lift-induced (or vortex) $\operatorname{drag} D_{i}$, and wave $\operatorname{drag} D_{w}$ :

$$
D_{\mathrm{ff}}:=D_{v}+D_{i}+D_{w}
$$

The method relies on the definition of a viscous volume and a shock-wave volume that is achieved based on physical criteria. This approach has the advantage of providing a physical breakdown of drag, which is of interest to the aircraft designer. On top of that, this method is capable of identifying spurious/numerical drag, thereby being more accurate,

$$
D_{\mathrm{sp}}:=D_{\mathrm{nf}}-D_{\mathrm{ff}}
$$

and the following overall equivalence is obtained:

$$
D_{f}+D_{p}=D_{v}+D_{i}+D_{w}+D_{\mathrm{sp}}
$$

These two well-tried methods will be used for comparison with the newly proposed exergy-based method introduced next.

\section{B. Exergy-Based Approach}

The formulation presented here is restricted to steady flows without energy addition, neither thermal nor mechanical, and its direct derivation can be found in [17].

The analysis is made in a continuous volume surrounding the body under study. The control volume is delimited by the aircraft surface $\mathcal{S}_{A}$, a surface excluding any discontinuous shock wave $\mathcal{S}_{w}$, and an outer boundary $\mathcal{S}_{O}$; see Fig. 1 . The latter is chosen so that the upstream and lateral surfaces are pushed far from the body and the downstream surface is a transverse plane perpendicular to the freestream located at a fixed distance downstream.

The exergy of the flow $\varepsilon$ is defined as the total enthalpy $h_{i}$ relative to the freestream minus the reference temperature $T_{\infty}$ times the entropy $s$ relative to the freestream,

$$
\varepsilon=\left(h_{i}-h_{i, \infty}\right)-T_{\infty}\left(s-s_{\infty}\right)
$$

which is a mathematical expression of exergy = energy - anergy. We are interested in the time-averaged change of this quantity within the control volume that can be expressed locally as

$$
\nabla \cdot(\rho \varepsilon \boldsymbol{V})=\nabla \cdot\left(\rho \delta h_{i} \boldsymbol{V}\right)-T_{\infty} \nabla \cdot(\rho \delta s \boldsymbol{V})
$$

Integrating this relation within the control volume before invoking the mass and momentum conservation as well as the first and second

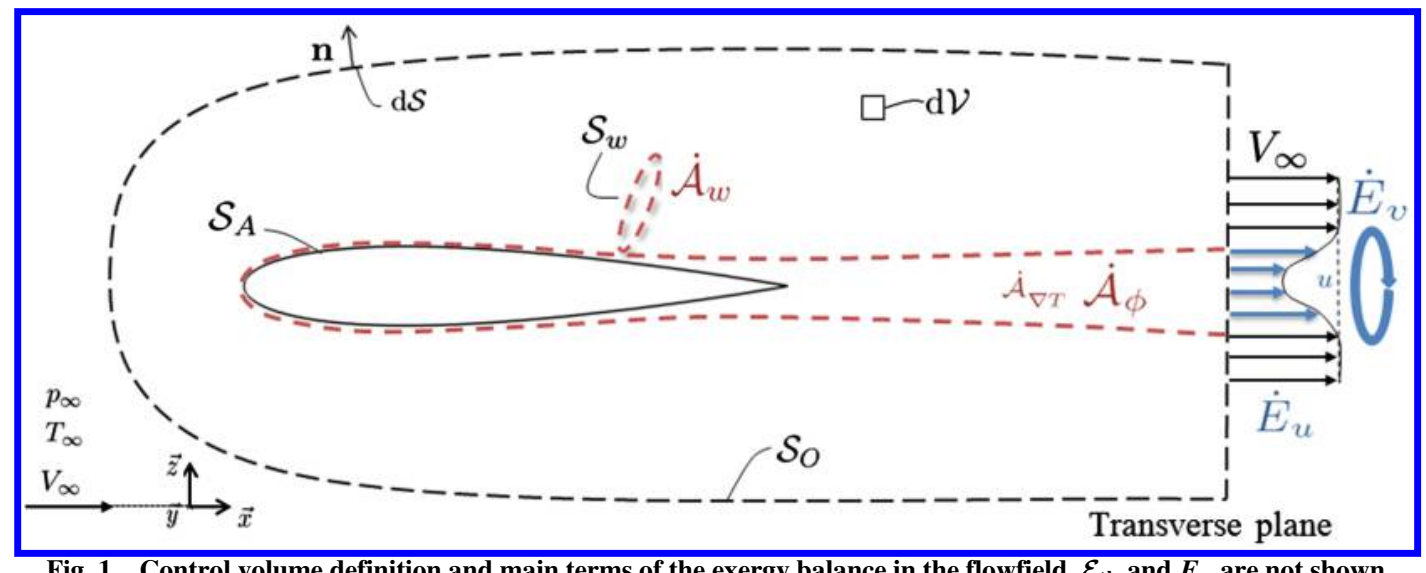

Fig. 1 Control volume definition and main terms of the exergy balance in the flowfield. $\mathcal{E}_{\text {th }}$ and $E_{p}$ are not shown. 
law of thermodynamics, one obtain the following drag-power relation:

$$
D V_{\infty}=\dot{\mathcal{E}}_{m}+\dot{\mathcal{E}}_{\text {th }}+\dot{\mathcal{A}}_{\phi}+\dot{\mathcal{A}}_{\nabla T}+\dot{\mathcal{A}}_{w}
$$

All terms are described hereafter and sketched in Fig. 1.

The mechanical exergy outflow:

$$
\begin{aligned}
\dot{\mathcal{E}}_{m} & :=\underbrace{\int_{\mathcal{S}_{o}} \frac{1}{2} \rho u^{2}(\boldsymbol{V} \cdot \boldsymbol{n}) \mathrm{d} \mathcal{S}}_{\dot{E}_{u}}+\underbrace{\int_{\mathcal{S}_{o}} \frac{1}{2} \rho\left(v^{2}+w^{2}\right)(\boldsymbol{V} \cdot \boldsymbol{n}) \mathrm{d} \mathcal{S}}_{\dot{E}_{v}} \\
+ & \underbrace{\int_{\mathcal{S}_{O}}\left(p-p_{\infty}\right)\left[\left(\boldsymbol{V}-\boldsymbol{V}_{\infty}\right) \cdot \boldsymbol{n}\right] \mathrm{d} \mathcal{S}}_{\dot{E}_{p}}
\end{aligned}
$$

$\dot{E}_{u}$ is the streamwise kinetic energy deposition rate and is associated with jet/wake, and $\dot{E}_{v}$ is the transverse kinetic energy deposition rates and is mainly associated with lift-induced vortices. $\dot{E}_{p}$ is a boundary pressure-work rate associated with these flow phenomena. All three terms being linked by velocity/pressure exchanges, they are gathered to form the rate of mechanical exergy outflow.

The thermal exergy outflow:

$$
\dot{\mathcal{E}}_{\text {th }}:=\int_{\mathcal{S}_{O}}\left[\rho\left(\delta e-T_{\infty} \delta s\right)+p_{\infty}\right](\boldsymbol{V} \cdot \boldsymbol{n}) \mathrm{d} \mathcal{S}
$$

Assuming a perfect gas, internal energy is solely proportional to temperature $\left(\delta e=c_{v} \delta T\right)$, so the first term is the rate of thermal energy outflow. The second term is the outflow rate of anergy, and the last term is usually referred to as the rate of (isobaric) surroundings work $[26,27]$ and is a nonavailable work that the system performs, or receives, due to its interaction with the reference atmospheric pressure field at $p_{\infty}$ [28]. The combination of the three terms yields the maximum amount of work that is theoretically extractable from the thermal energy.

The viscous anergy:

$$
\dot{\mathcal{A}}_{\phi}:=\int_{\mathcal{V}} \frac{T_{\infty}}{T} \Phi_{\text {eff }} \mathrm{d} \mathcal{V}
$$

where $\Phi_{\text {eff }}=\left(\mu+\mu_{t}\right) \overline{\bar{S}}$ with $\overline{\bar{S}}$ being the mean rate of strain tensor and $\mu_{t}$ being the eddy viscosity. $\dot{\mathcal{A}}_{\phi}$ is linked to viscous dissipation and turbulence, which transform any difference in kinetic energy into thermal energy. For that reason, these phenomena act toward reducing the mechanical exergy to zero by building a new homogeneous field of velocity (and pressure), in which the system and its surroundings are back in mechanical equilibrium. The viscous dissipation can be expressed as a function of the square of the velocity gradients. This term is positive and hence will always increase anergy; it indicates an irreversible process that destroys mechanical exergy.

The thermal anergy:

$$
\dot{\mathcal{A}}_{\nabla T}:=\int_{\mathcal{V}} \frac{T_{\infty}}{T^{2}} k_{\text {eff }}(\nabla T)^{2} \mathrm{~d} \mathcal{V}
$$

where $k_{\text {eff }}=c_{p}\left(\mu / P r+\mu_{t} / P r_{t}\right)$ with $P r$ and $P r_{t}$ being the Prandtl and turbulent Prandtl numbers, respectively. $\dot{\mathcal{A}}_{\nabla T}$ is linked to thermal mixing, which reduces the difference in temperature and therefore reduces the thermal exergy to zero. It acts toward building a new homogeneous field of temperature in which the system and its surroundings are back in thermal equilibrium. The thermal mixing is a function of the square of the temperature gradients so that avoiding strong temperature variations would reduce this term. This term is positive and hence will always increase anergy; it indicates an irreversible process that destroys thermal exergy.

The wave anergy:

$$
\dot{\mathcal{A}}_{w}:=T_{\infty} \int_{\mathcal{S}_{w}} \rho \delta s(\boldsymbol{V} \cdot \boldsymbol{n}) \mathrm{d} \mathcal{S}
$$

This phenomenon converts mechanical energy into thermal energy; this process being irreversible, it is an exergy destruction. The computation if this term relies on the definition of a shock surface $S_{w}$ enclosing the entropy associated with this flow phenomenon.

As we move away from the body, the mechanical exergy $\dot{\mathcal{E}}_{m}$ and thermal exergy $\dot{\mathcal{E}}_{\text {th }}$ outflows are gradually dissipated by generation of viscous anergy $\dot{\mathcal{A}}_{\phi}$ and thermal anergy $\dot{\mathcal{A}}_{\nabla T}$, respectively. Additional losses are associated with the presence of a shock wave $\dot{\mathcal{A}}_{w}$. Within the exergy/anergy framework, aerodynamic losses are defined as "the reduction in total vehicle work potential due to irreversible fluiddynamic interactions between the vehicle and the atmosphere" [29]. In the (very) far field of the solid, the rate of work performed by drag $D V_{\infty}$ is equal to the total anergy generated, i.e., the total entropy times the reference temperature. This statement was first made by Oswatitsch in 1956 [30].

Equation (7) can be considered as an extension to transonic flows of the exergy-based approach to incompressible flows of Li et al. [15] and Monsch et al. [16]. In addition, this relation is (theoretically) equivalent to the restriction to unpowered airframe cases of the mechanical energy balance proposed by Drela [31]. It has also been demonstrated that many (past and current) momentum-based farfield drag expressions [22,32-36] required some thermodynamic considerations and that their underlying theory can be consistently related to the proposed exergy-based formulation []].

\section{Numerical Implementation}

ONERA has much experience in the prediction and breakdown of the drag from numerical flow solutions via its far-field drag method. Several years ago, the approach developed by Destarac [24] and van der Vooren and Destarac [25] was numerically implemented in a postprocessing code named $f f d$ for far-field drag. More recently, a new code named $f f x$ (for far-field exergy) was developed on the basis of this existing code for the assessment of the aerothermopropulsive performance of complex configurations based on the formulation derived in [8]. As illustrated in Fig. 2, it is a postprocessing code, meaning that its input data comes from a computational fluid dynamics (CFD)-RANS solver.

In a nutshell, the flow solution computed from the CFD solver is extracted and represents the input data for the postprocessing code, which recalculates any quantity of interest (like entropy) and subsequently gives the user the required information for the assessment of the performance of the configuration from an exergy point of view. For RANS flow solutions, the numerical implementation is based on the model for mean entropy production proposed by Moore and Moore in 1983 [37]. This model greatly lessens the issues encountered by Reynolds-averaging the entropy transport equation. However, as it has only been used to qualitatively assess the aerodynamic performance of a few unpowered aerospace configurations [12-16], the implementation of this model requires a detailed validation. The code has a few tuning options such as an automatic wake analysis whereby the distance between the body and the transverse plane is gradually increased, the corresponding values of all terms of Eq. (7) being written in an output file.

Finally, all terms of Eq. (7) are expressed in terms of power counts, abbreviated pc, like for the viscous anergy production:

$$
C \dot{\mathcal{A}}_{\phi}=\frac{\dot{\mathcal{A}}_{\phi}}{0.5 \rho_{\infty} V_{\infty}^{3} A_{\text {ref }}}
$$

By doing so, their magnitudes are directly comparable to the more traditional drag coefficients expressed in drag counts, abbreviated dc.

\section{Results and Discussion}

The objective of this test case is to validate the formulation and its numerical implementation for unpowered configurations. This validation is made with reference to experimental data and to the two previously described near-field and far-field drag methods. 


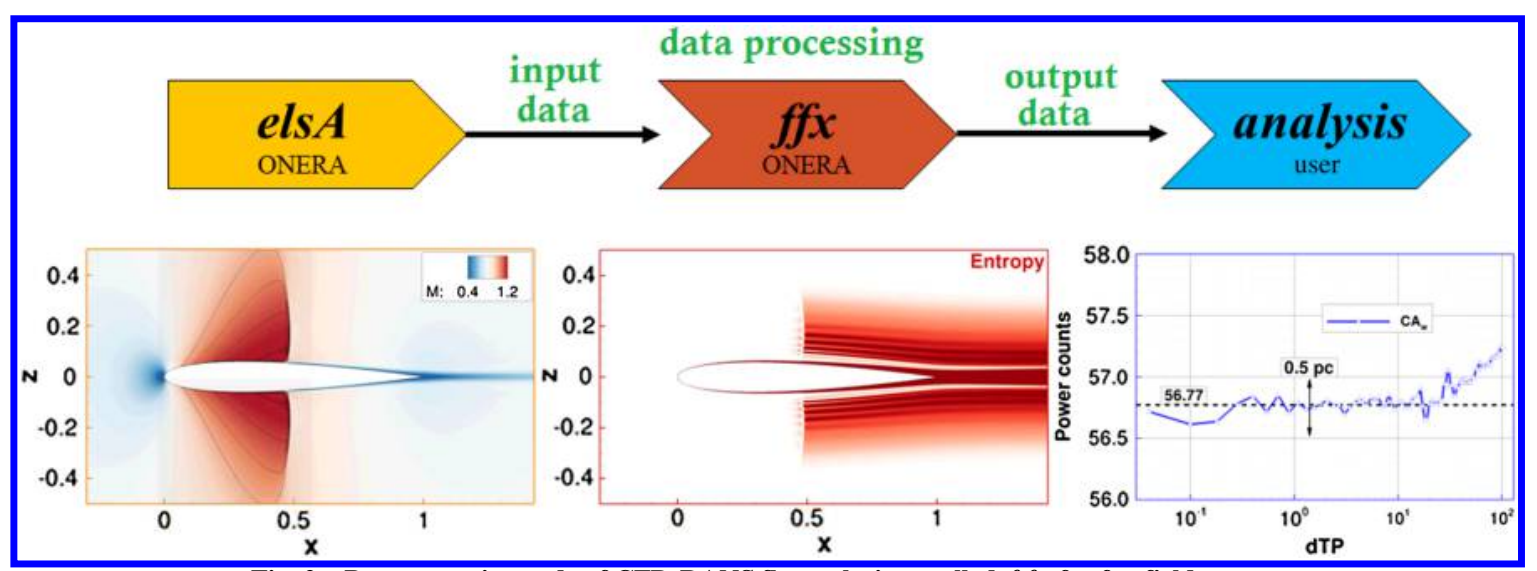

Fig. 2 Postprocessing code of CFD-RANS flow solutions called $f f x$ for far-field exergy.

\section{A. NASA Common Research Model}

\section{Context}

The Drag Prediction Workshop (DPW) series was initiated in 2001 by a working group of the AIAA Applied Aerodynamics Technical Committee. The first objective was to assess the state-of-the-art computational methods as practical aerodynamic tools for aircraft drag and moment prediction. Over the years, it has provided an impartial forum for evaluating the effectiveness of existing CFD codes and modeling techniques using Navier-Stokes solvers.

The detailed aerodynamic design of the Common Research Model (CRM) used for the fifth DPW workshop was performed by teams from The Boeing Company [38]. The reference geometry is characterized by a mean aerodynamic chord (MAC) of $c=7.00 \mathrm{~m}$, a reference surface area of $383.68 \mathrm{~m}^{2}$ (full model), and a semispan of $b=29.38 \mathrm{~m}$ for an aspect ratio $A R=9.0$. The general characteristics of the geometry are representative of a Boeing B777-200 without vertical and horizontal stabilizers and without engines; see Fig. 3 .

Case $\overline{1}$ of the Fifth Drag Prediction Workshop (2012) is a gridconvergence study on the prediction of the drag experienced by the wing-body configuration in cruise (transonic) conditions: $M_{\infty}=$ 0.85 and $C L=0.5$ [21]. The Reynolds number was chosen to be in agreement with wind-tunnel conditions, $R e_{c}=510^{6}$. To obtain a reference value independent of any numerical considerations, a windtunnel model was fabricated and tested in the National Transonic Facility (NTF) in Hampton, VA, [39] and at the NASA Ames Research Center at Moffett Field, CA [40].

\section{Numerical Considerations}

a. Grids. To allow participation as large as possible, the DPW Committee provided a family of six point-matched multiblock and structured grids ranging from 0.6 to 138 million elements; see Table 1. It was built from an extrafine mesh of 41 million elements (L5) from which a superfine mesh of 138 million hexahedra (L6) was created by increasing the numbers of cells in each direction by a factor of 1.5, while maintaining grid distribution. These two grids were then coarsened twice, consistent with the multigrid technique, to obtain a multiblock family of six self-consistent grids.

The sizes of these grids therefore range from 0.64 to 138.02 million hexahedra, exhibiting a grid-size ratio of 216. These meshes are O-type grids and have been created by extrusion of the surface discretization and are made of five structured blocks. Through this strategy, a precise control on grid quality, such as grid spacing, stretching ratio, and grid orthogonality near configuration surfaces, is achieved; see Figs. $\underline{4}$ and $\underline{5}$. The $y^{+}$ranges from 2.00 for the tiny grid to 0.33 for superfine mesh. All grids exhibit an extension greater than 100 mean-aerodynamic chords in all directions. Note the large range of refinement levels provided by the grid family provided by the DPW Committee, which is perfectly suited for an extensive gridconvergence study.

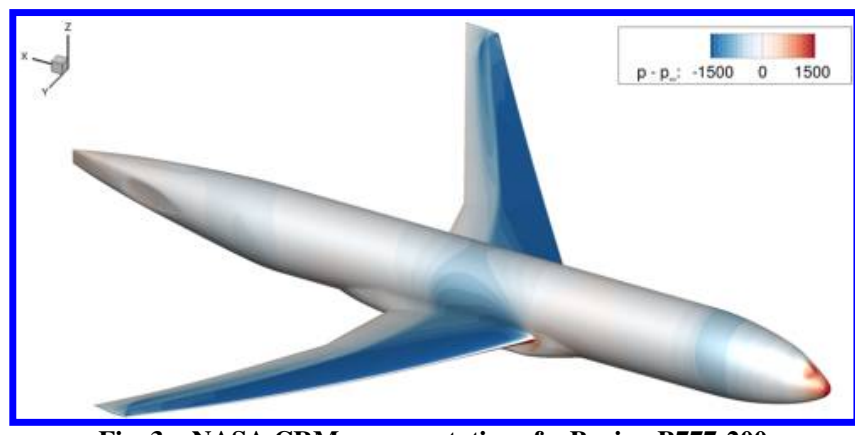

Fig. 3 NASA CRM representative of a Boeing B777-200.

b. Computation of Flow Solutions. Structured RANS computations were performed with the ONERA-elsA solver [41]. This software was used here with a cell-centered finite-volume discretization. Time integration was carried out by a backward Euler scheme with implicit LU-SSOR relaxation. Spatial discretization was realized using a second-order accurate central Jameson scheme with artificial viscosity [42]. Depending on the grid, multigrid techniques were used to accelerate the convergence. All of the flow solutions were set as fully turbulent ${ }^{\ddagger}$ with the Spalart-Allmaras turbulence model [43]. A target lift algorithm enabled reaching the requested accuracy of the lift coefficient $(C L=0.5 \pm 0.001)$ for all grids; the corresponding incidence ranged from 2.150 to $2.227 \mathrm{deg}$; see Table 1 . In terms of convergence, the computations were continued until the near-field fluxes were stable enough to observe a lift and drag variation lower than 0.001 and 0.0001 (one drag count), respectively [44]. All these computations were realized with CGNS input and output files.

\section{Momentum-Based Drag Methods}

Before analyzing the flow from an exergy point of view, the drag prediction from the near-field and far-field methods enables an assessment of the flow solutions. The key relations are

$$
\begin{gathered}
D_{\mathrm{nf}}=D_{p}+D_{f}, \quad D_{\mathrm{ff}}=D_{v}+D_{i}+D_{w}, \\
D_{\mathrm{sp}}=D_{\mathrm{nf}}-D_{\mathrm{ff}}
\end{gathered}
$$

Table $\underline{2}$ presents the near-field and far-field drag decomposition from the $f f d 72$ code.

Friction drag $C D_{f}$ is underestimated on coarse meshes, while pressure drag $C D_{p}$ is overestimated. The total near-field $C D_{\mathrm{nf}}$ varies very little for the three finest meshes, around $249.8 \mathrm{dc}$. The far-field drag approach enables a physical breakdown of drag into a viscous

The flow starts out laminar, and the location where it becomes fully turbulent is dependent on the turbulence model and grid density. 
Table 1 Characteristics of the six multiblock structured grids provided by the DPW Committee, as well as $\alpha$ and $C L$

\begin{tabular}{lccccc}
\hline \hline Level & Name & Elements (millions) & $y^{+}$ & $\alpha$ & $C L$ \\
\hline L1 & Tiny & 0.64 & 2.00 & $2.227^{\circ}$ & 0.5001 \\
L2 & Coarse & 2.16 & 1.33 & $2.181^{\circ}$ & 0.4995 \\
L3 & Medium & 5.11 & 1.00 & $2.169^{\circ}$ & 0.5003 \\
L4 & Fine & 17.25 & 0.67 & $2.150^{\circ}$ & 0.4993 \\
L5 & Extrafine & 40.89 & 0.50 & $2.153^{\circ}$ & 0.5002 \\
L6 & Superfine & 138.02 & 0.33 & $2.153^{\circ}$ & 0.5004 \\
\hline \hline
\end{tabular}

drag $C D_{v}$, a (lift-)induced drag $C D_{i}$, and a wave drag $C D_{w}$. One can see that this latter component is very small, around $4 \mathrm{dc}$, which represents $2 \%$ of the total aircraft drag. The lift-induced component accounts for $90 \mathrm{dc}(62 \%)$, while the remaining $155 \mathrm{dc}(36 \%)$ are associated with viscous phenomena. Each component appears well converged with grid refinement. Refer to [44] for a more detailed analysis of this test case with the far-field drag method.

The far-field drag formulation implemented in $f f d 72$ is also capable of discriminating the spurious (numerical) drag $C D_{\mathrm{sp}}$, which is below $1 \mathrm{dc}$ starting from the medium grid (L3). The overall good agreement between the near-field and far-field drag methods suggests trustworthy flow solutions in terms of numerics. This test case is a perfect candidate to assess the pertinence of the exergybased formulation as well as the robustness and accuracy of the postprocessing code $f f x$. A flow analysis from an exergy standpoint is introduced next before applying the approach in terms of drag prediction.

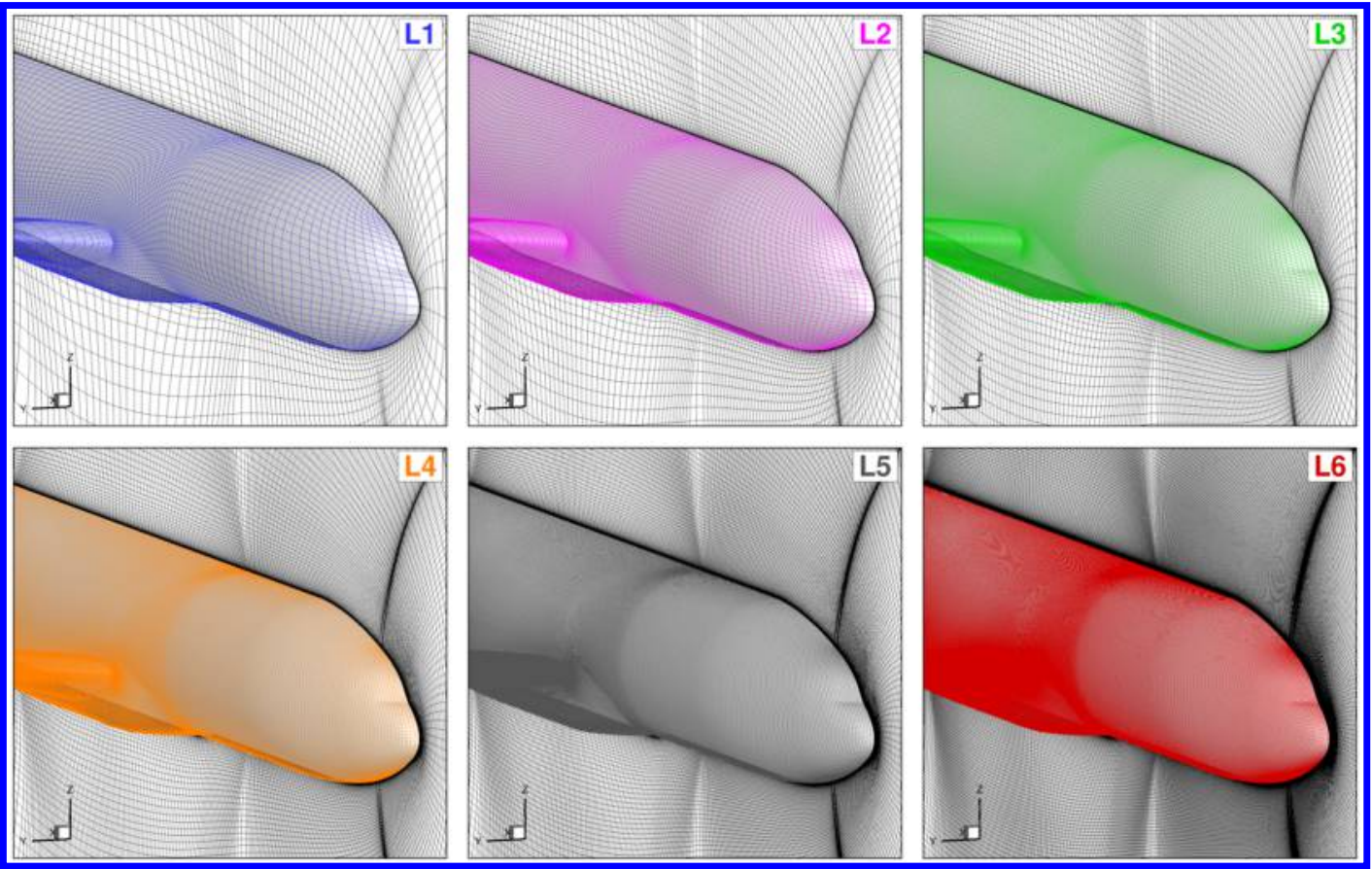

Fig. 4 Surface and symmetry plane spatial discretization, zoom on the nose.
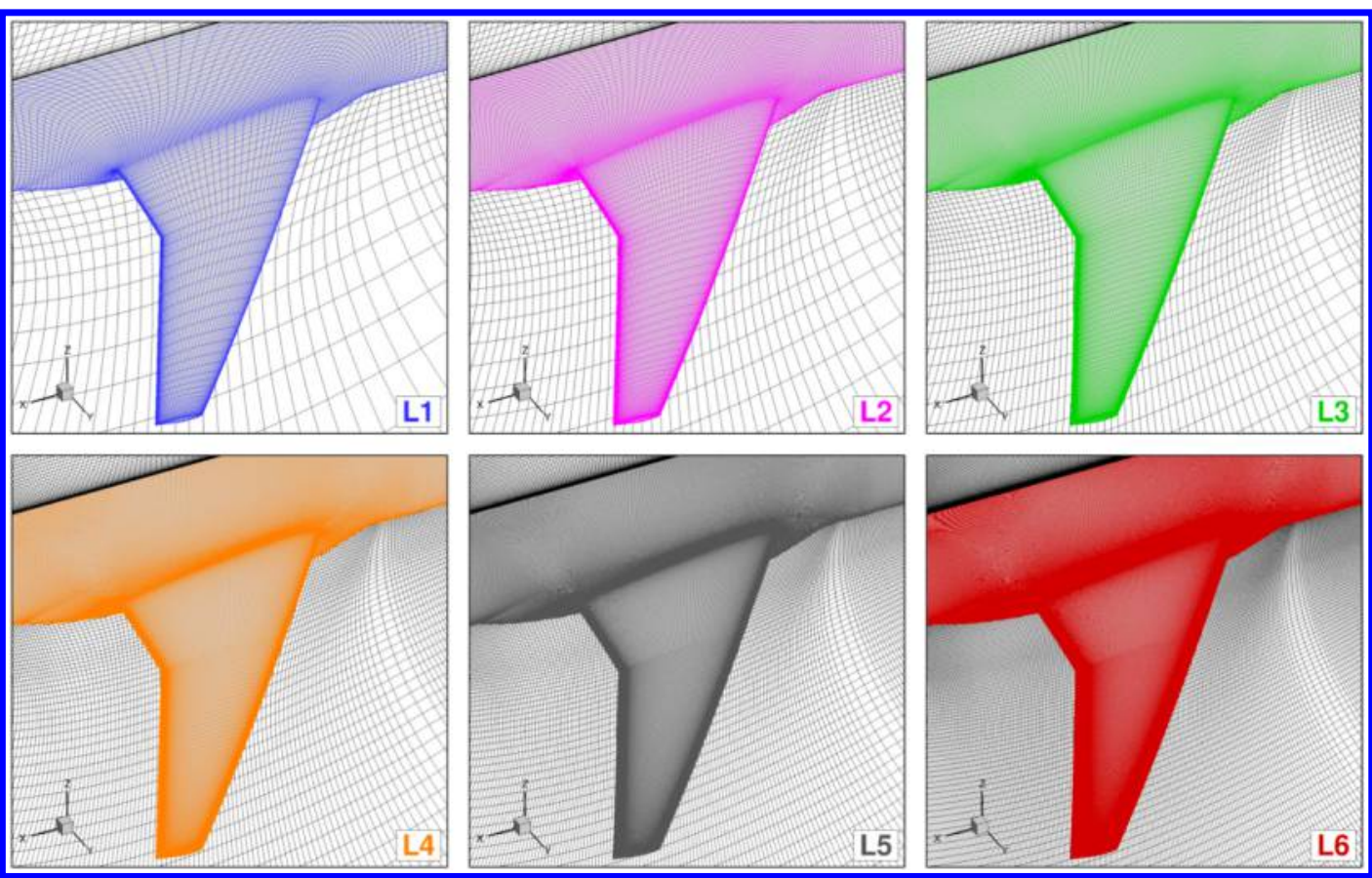

Fig. 5 Surface and symmetry plane spatial discretization, zoom on the wing-body junction. 
Table 2 Near-field and far-field drag components from $f f d 72$ with mesh refinement

\begin{tabular}{lccccccccc}
\hline \hline & \multicolumn{3}{c}{ Near-field drag } & & \multicolumn{5}{c}{ Far-field drag } \\
\cline { 2 - 4 } \cline { 7 - 8 } Mesh & $C D_{f}$ & $C D_{p}$ & $C D_{\mathrm{nf}}$ & & $C D_{v}$ & $C D_{i}$ & $C D_{w}$ & $C D_{\mathrm{ff}}$ & $C D_{\text {sp }}$ \\
\hline L1 & 113.5 & 146.2 & 259.7 & & 159.0 & 91.5 & 4.8 & 255.3 & 4.4 \\
L2 & 113.8 & 139.2 & 253.0 & & 156.4 & 90.9 & 3.9 & 251.2 & 1.8 \\
L3 & 114.1 & 137.3 & 251.4 & & 155.9 & 90.9 & 3.8 & 250.6 & 0.8 \\
L4 & 114.6 & 135.2 & 249.8 & & 155.4 & 90.4 & 3.7 & 249.5 & 0.3 \\
L5 & 114.8 & 135.1 & 249.9 & & 155.2 & 90.6 & 3.8 & 249.6 & 0.3 \\
L6 & 115.0 & 134.8 & 249.8 & & 155.2 & 90.6 & 3.9 & 249.7 & 0.1 \\
\hline \hline
\end{tabular}

\section{B. Flowfield Analysis}

A qualitative and quantitative flow analysis is made on the finest mesh (L6), first in terms of exergy outflows and then in terms of anergy generation.

\section{Recoverable Exergy}

One important aspect of the exergy analysis is to pinpoint any source of improvement in a fluid flow [8]. The object, here the aircraft, has generated perturbations in terms of velocity, pressure, and temperature, which, in order to satisfy Second Law requirements, have to be dissipated. Until these deviations from the reference conditions are damped, they represent a potential for improvement because they could be valued. The exergy-waste coefficient (EWC) can be introduced to quantify the relative amount of exergy that is being lost,

$$
\mathrm{EWC}=\frac{\dot{\mathcal{E}}_{\mathrm{rec}}}{\dot{\mathcal{E}}_{\mathrm{rec}}+\dot{\mathcal{A}}_{\mathrm{tot}}}=\frac{\dot{\mathcal{E}}_{m}+\dot{\mathcal{E}}_{\mathrm{th}}}{D V_{\infty}}
$$

where the recoverable exergy $\dot{\mathcal{E}}_{\text {rec }}$ is made up of a mechanical part $\dot{\mathcal{E}}_{m}$ and a thermal part $\dot{\mathcal{E}}_{\text {th }}$. Figure 6a gives the evolution of this coefficient downstream of the aircraft up to a distance of about 80 mean aerodynamic chords. At a distance of 70 mean aerodynamic chords (or 8 fuselage lengths, $\approx 500 \mathrm{~m}$ ), the $\mathrm{EWC}_{70} \approx 33 \%$ represents around $2 \mathrm{MW}$ for a complete aircraft. $\mathrm{s}$ Because the flow analysis at such a large distance from the body involves some uncertainty, even on the finest grid available, the value of $2 \mathrm{MW}$ should be considered as an order of magnitude. This estimation represents the absolute amount of mechanical work that could be extracted and does not involve any particular system to achieve this recovery.

From Fig. 6 b, one can see that the recoverable exergy is mainly in the mechanical form with a thermal exergy outflow therefore rapidly vanishing. As a consequence, the transverse kinetic energy, associated with the presence of the lift-induced vortices, see Fig. 7, remains the main contributor far from the aircraft. This potential could be partially recovered by turbines mounted on the wingtips [45,46], while formation flight [47] could take advantage of this transverse kinetic energy in the far field of the airplane.

This commonly accepted example of recoverable loss is well captured by the present methodology and has been extended to the quantification of the work potential present in the wake/jet downstream of an engine [18].

\section{Anergy Generation}

The anergy field is depicted in Fig. $\underline{8}$ by the wave surface and by isosurfaces (of same level) of thermal and viscous anergy. The threshold value chosen to define the isosurfaces is lower on the left and bottom figures.

First, from the bottom picture, we can see that the isosurfaces associated with the dissipation in the wake remains several fuselage lengths downstream of the aircraft, up to the mesh boundaries. This observation is valid on the finest mesh, but on a coarser grid, these isosurfaces decline more rapidly because the numerical dissipation

\footnotetext{
${ }^{\S}$ The recoverable exergy is given by $(1 / 2) \rho_{\infty} V_{\infty}^{3} C \dot{\mathcal{E}}_{\text {rec }} A_{\text {ref }}$, which, with $C \dot{\mathcal{E}}_{\text {rec }} \approx 82 \mathrm{pc}, \rho_{\infty}=0.045 \mathrm{~kg} \cdot \mathrm{m}^{3}, V_{\infty}=300 \mathrm{~m} \cdot \mathrm{s}^{-1}$ and $A_{\text {ref }}=384 \mathrm{~m}^{2}$, yields $2 \mathrm{MW}$.
}

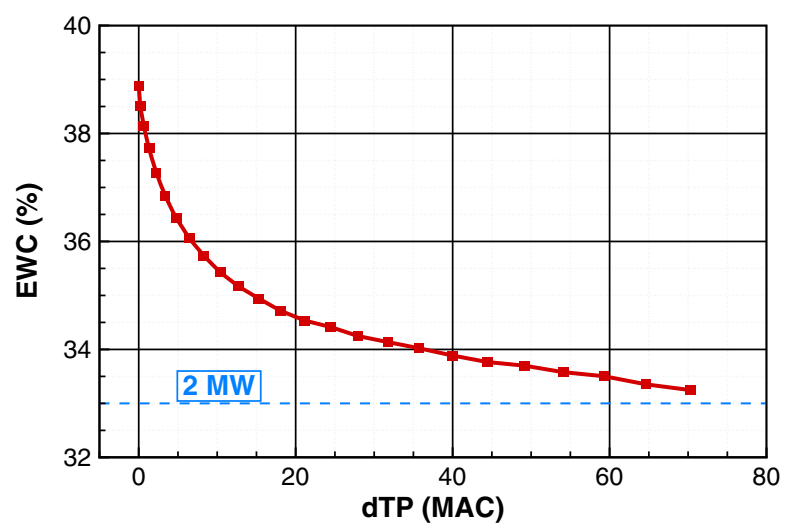

a) Exergy-waste coefficient

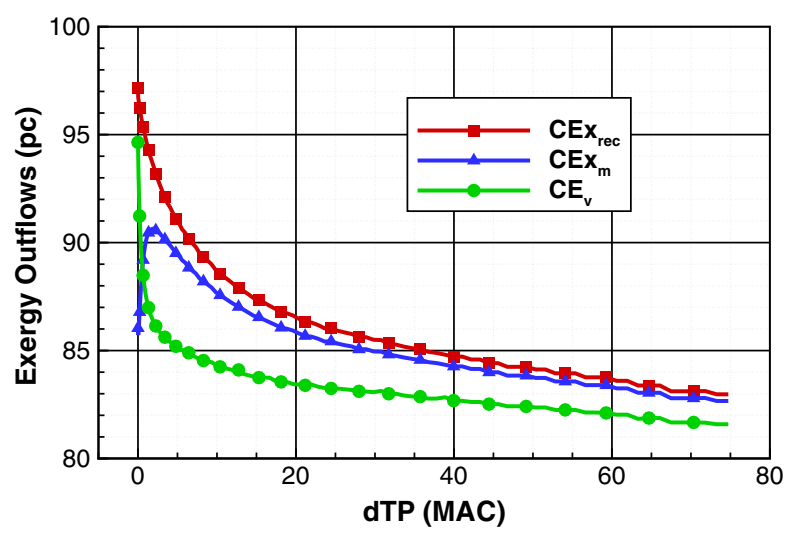

b) Decomposition of the recoverable exergy

Fig. 6 Recoverable exergy in the wake, superfine mesh (L6).

overtakes the physical dissipation. The thermal anergy is mostly generated in the wake of the fuselage, while the viscous dissipation is clearly connected to lift-induced vortices; the rollup of the shear layer is clearly visible on the left picture. From the left picture, we distinguish the formation of shock waves, with a small contribution above the fuselage. The shock pattern at the wing tip is highlighted in the middle zoom.

Quantitatively speaking, Fig. 9 gives the streamwise and spanwise rate of change of anergy generation with a representation of the aircraft and of the shock surface.

As regards the streamwise distribution, one can first notice a zone just downstream of the nose that corresponds to the dissipation of the perturbations due to the penetration of the body in the fluid. Then, the predominant source of anergy generation appears to be the wing; the rates rapidly fall toward the wake for all dissipative phenomena. As regards the spanwise distribution, the fuselage involves extremely high rates of anergy generation (above scale) that rapidly reduce over the wing. The total anergy presents bumps (relative to the viscous + thermal anergy) that are directly related to the three (separate) shocks that are visible on the wing; see Fig. $\underline{8}$. This quantitative assessment gives the aircraft designer the magnitude of the losses associated with each dissipative phenomenon and is complementary to the flow visualization.

On both distributions, viscous anergy is predominant over the thermal anergy, which is in agreement with the qualitative assessment of the Boeing B747-200 in cruise conditions of Alabi et al. [12]. As the Mach number increases, the thermal dissipation rises but never exceeds about $10 \%$ in the transonic regime [19].

This visualization of the flow from an anergy point of view enables the aircraft designer to pinpoint the location of the losses in the flowfield. By doing so, regions of potential improvement are highlighted, and the impact of modifying the configuration can be visually assessed. For certain architectures, like blended-wing bodies, aerodynamic losses cannot be defined as (viscous, wave, and induced) drag in a satisfactory manner because drag itself cannot be 


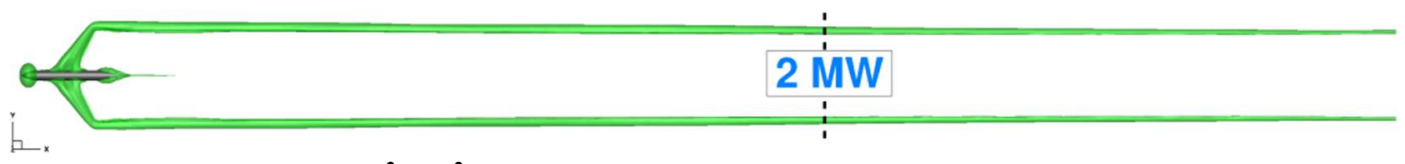

Fig. 7 Isosurface of transverse kinetic energy $\left(v^{2}+w^{2}\right)$ and work potential evaluated 8 fuselage lengths downstream of the airplane, superfine mesh (L6).

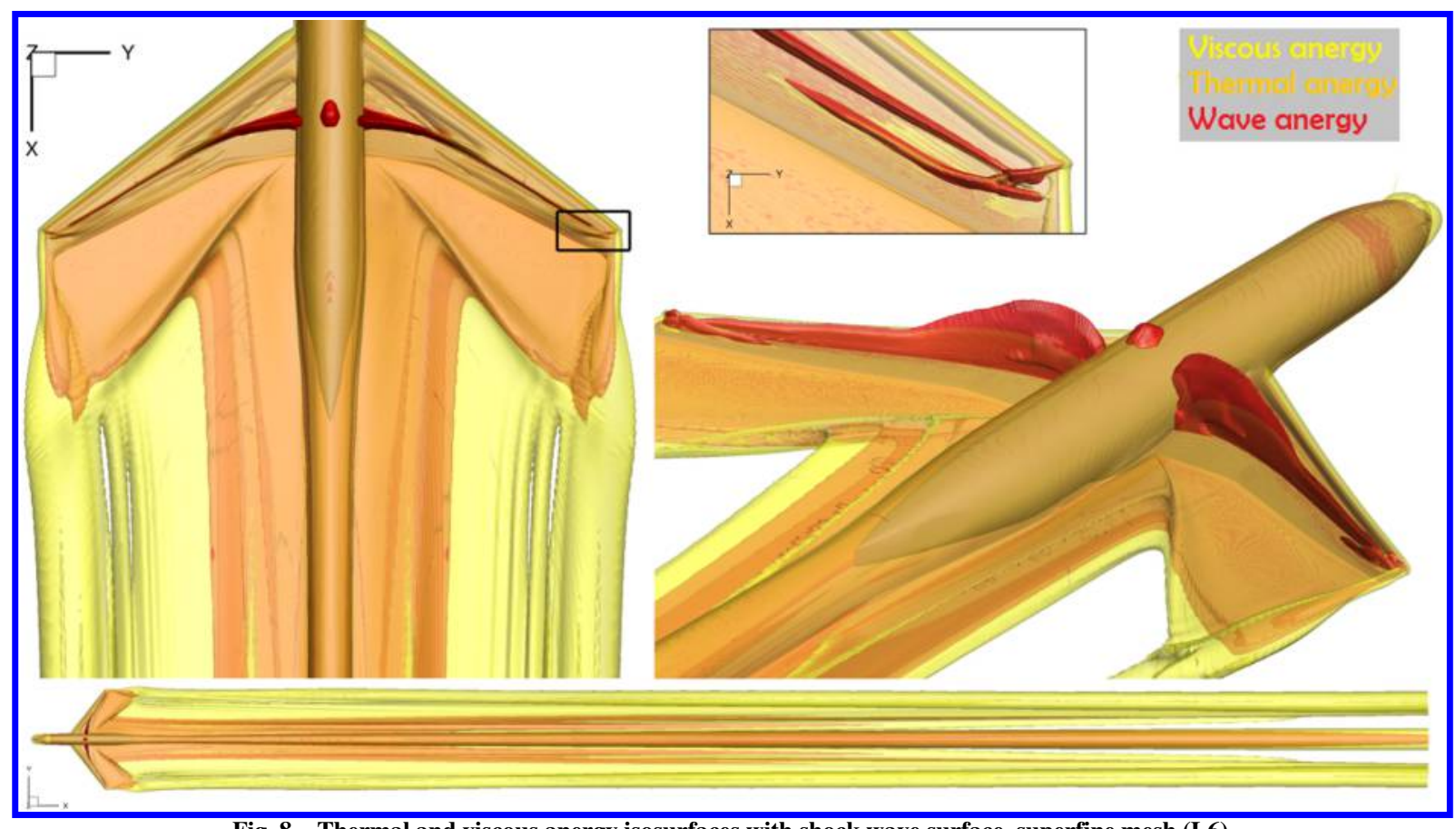

Fig. 8 Thermal and viscous anergy isosurfaces with shock wave surface, superfine mesh (L6).
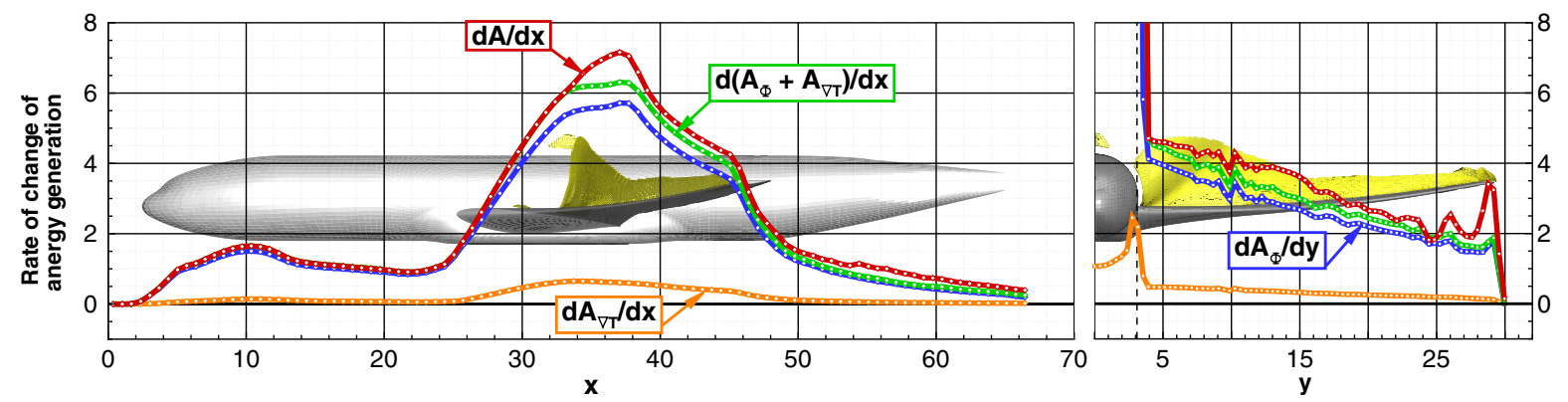

Fig. 9 Rate of change of viscous, thermal, and total anergy generation. Left: chordwise distribution, right: spanwise distribution, superfine mesh (L6).

easily defined [5]. The proposed methodology presents the advantage of providing a global accounting and phenomenological breakdown [20].

\section{Grid Convergence Study on Drag Prediction}

To determine the sensitivity of the code to mesh density, a grid convergence study is conducted by first defining a generic control volume and then defining the shock surface. An analysis of the wake shows that a correction of the calculation of the viscous and thermal anergy generation is required. Finally, the efficiency of the proposed correction is highlighted for an accurate drag prediction, and a few comments are made regarding its benefits.

\section{Generic Control Volume Definition}

For the present study, the control volume is custom made and based on airplane geometric considerations. Figure 10 gives an overview for the medium mesh (L3).

The left-hand-side picture represents the computational volume with the $X-Z$ plane being the symmetry plane of the aircraft. The upstream and lateral surfaces of the outer boundary are pushed up to the mesh boundaries so that the control volume is delimited by the transverse plane; all fluid elements upstream of this plane are tagged as being part of the control volume. The rest of the mesh volume is outside of the control volume. The right-hand-side pictures give more information about the transverse plane in the vicinity of the configuration. It is defined by tagging each element of which the $x$ coordinate is greater than a datum value. The transverse plane is close to the aircraft (low $d_{\mathrm{TP}}$ ) in order to avoid the spurious dissipation of the vortices. Its distance from the airplane is determined next, in agreement with considerations on the accuracy of the total drag prediction.

Also there is no need to rely on any viscous (or thermal) volume; the integration of this term is made in the entire control volume.- By doing so, we avoid the need for a viscous volume that is usually required in far-field drag methods. The definition of this volume

\footnotetext{
TThe circular rings observable in the figure on the top right are due to the topology of the grid.

**As shown in Fig. $\underline{8}$, regions of great contributions are directly highlighted.
} 


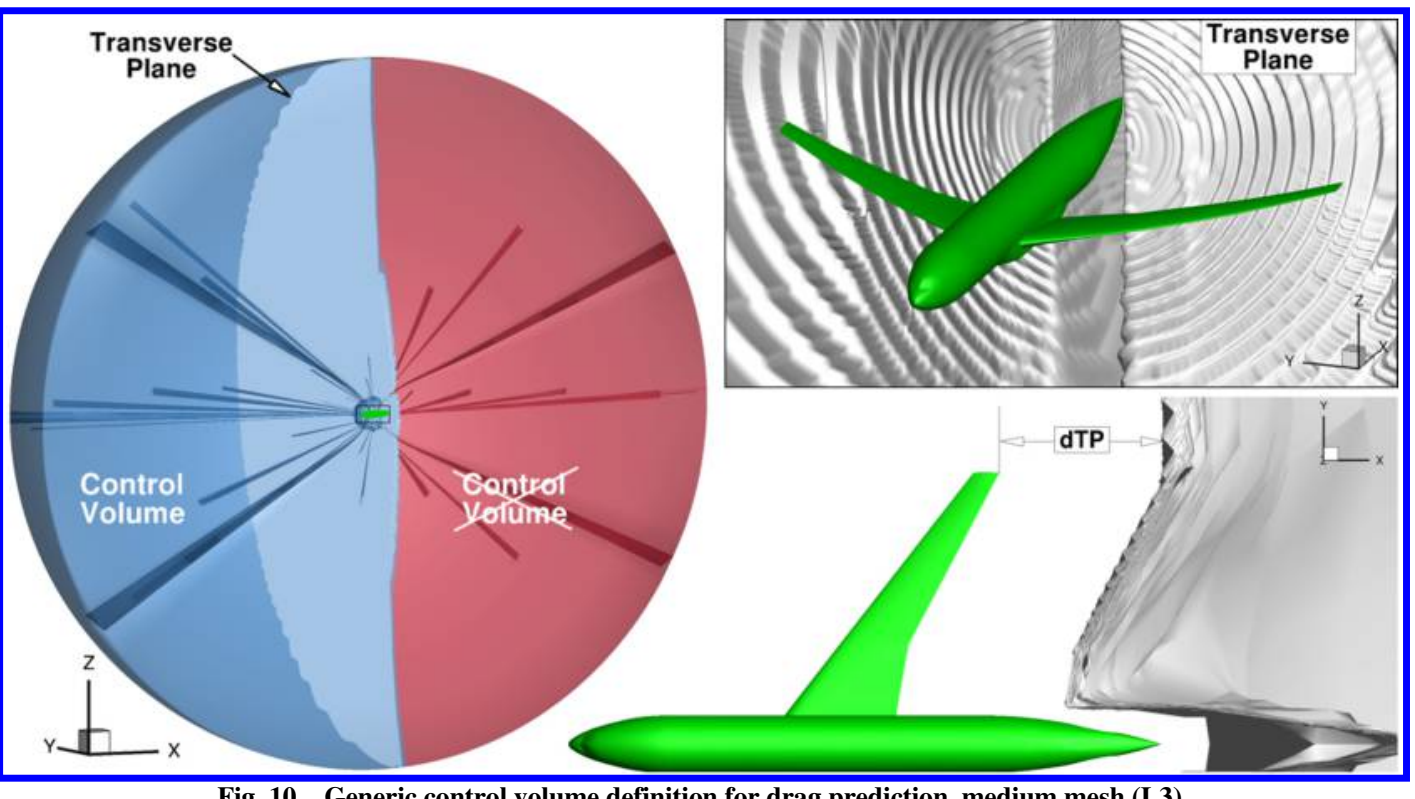

Fig. 10 Generic control volume definition for drag prediction, medium mesh (L3).

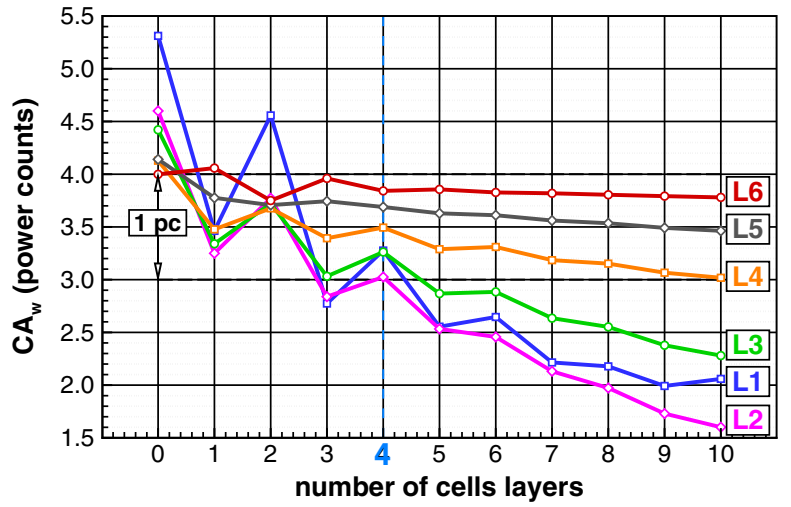

Fig. 11 Wave anergy vs the number of cell layers, with grid refinement.

relies on physical sensors but is not unique and must be calibrated $[\underline{24}, \underline{34}, \underline{35}, \underline{48}]$, just like the shock surface described next.

\section{Shock Wave Treatment}

The numerical treatment of the shock wave relies on the definition of a surface enclosing the entropy associated with this irreversible phenomenon. Most far-field drag methods also require such a surface to isolate this flow feature. The method implemented in $f f d 72$ to define such a surface relies on a shock detector introduced by Lovely and Haimes in 1999 [49]:

$$
\lambda_{w}=\frac{V \cdot \nabla p}{a\|\nabla p\|}
$$

If $\lambda_{w}$ is greater than a threshold value, the cell under consideration is tagged as being enclosed by the surface. From existing experience, a datum value of 0.95 is chosen. Yet the initial guess from this detector is not always the most adapted, and it has been found that the addition of a few cell layers provides more accurate results. The precise number of layers is mesh and user dependent; there is not a universal value. Figure 11 gives the evolution of the wave anergy coefficient with an increasing number of cell layers and with mesh refinement.

The general observation is that the addition of a few layers smoothes the coefficient for all grid densities. One can also observe an oscillatory behavior of the coefficient that comes from the flow solution itself as highlighted in the slice at $y / b=54.5 \%$ in Fig. 12: regions of entropy increase are directly followed by regions of entropy reduction. On average, regions of entropy reduction are predominant, which explains the diminishing wave anergy coefficient as the number of layers increases.

As the grid is refined, the sensitivity of the wave anergy to the number of layers clearly reduces. For the present analysis, four cell layers are added to define the shock surface, but one could argue that a value of two or three cell layers could also have been chosen. Also note the close agreement among all grids close to $3.5 \mathrm{pc} \pm 0.5$, which is also in excellent agreement with the wave drag coefficients computed by the well-tried far-field drag method; see Table 2 .

From visual considerations in Fig. 12, one can infer that the boundary layers are well preserved. In fact, the viscous and thermal anergy integrated within the wave volume selected each account for less than $10^{-4} \mathrm{pc}$. As a consequence, the determination of the wave anergy has no impact on the other dissipative components, as opposed to some momentum-based approaches [35]. For these methods, the choice of the surface $\mathcal{S}_{w}$ involves some sharing between the viscous drag and the wave drag. This problem is completely absent in the present implementation of the exergy method, which represents a numerical advantage.

3. Wake Analysis in Terms of Anergy Generation and Exergy Destruction

Equation (7) is theoretically valid for any (closed) outer boundary $\mathcal{S}_{O}$ enclosing the body. As the transverse plane is moved away from the body, we scan the wake in terms of anergy generation and exergy destruction to evaluate the consistency of the theoretical balance,

$$
\begin{aligned}
\frac{\mathrm{d}}{\mathrm{d} x_{\mathcal{T P}}} D V_{\infty} & =\frac{\mathrm{d}}{\mathrm{d} x_{\mathcal{T P}}} \dot{\mathcal{E}}_{m}+\frac{\mathrm{d}}{\mathrm{d} x_{\mathcal{T P}}} \dot{\mathcal{E}}_{\mathrm{th}}+\frac{\mathrm{d}}{\mathrm{d} x_{\mathcal{T P}}} \dot{\mathcal{A}}_{\phi}+\frac{\mathrm{d}}{\mathrm{d} x_{\mathcal{T P}}} \dot{\mathcal{A}}_{\nabla T}+\frac{\mathrm{d}}{\mathrm{d} x_{\mathcal{T P}}} \dot{\mathcal{A}}_{w} \\
0 & =\frac{\mathrm{d}}{\mathrm{d} x_{\mathcal{T P}}} \dot{\mathcal{E}}_{m}+\frac{\mathrm{d}}{\mathrm{d} x_{\mathcal{T P}}} \dot{\mathcal{E}}_{\mathrm{th}}+\frac{\mathrm{d}}{\mathrm{d} x_{\mathcal{T P}}} \dot{\mathcal{A}}_{\phi}+\frac{\mathrm{d}}{\mathrm{d} x_{\mathcal{T} P}} \dot{\mathcal{A}}_{\nabla T}
\end{aligned}
$$

where we note that the rate of drag work and the wave anergy are independent on the transverse plane location. This relation can be rearranged into

$$
0=\frac{\mathrm{d}}{\mathrm{d} x_{\mathcal{T} \mathcal{P}}}\left(\dot{\mathcal{E}}_{m}+\dot{\mathcal{E}}_{\mathrm{th}}\right)+\frac{\mathrm{d}}{\mathrm{d} x_{\mathcal{T P}}}\left(\dot{\mathcal{A}}_{\phi}+\dot{\mathcal{A}}_{\nabla T}\right)
$$

In other words, the rate of decrease in exergy outflows should match the rate of anergy generation. Figure 13 gives the necessary information to assess the numerical (in)consistency of this relation in the wake of the body. The abscissa is expressed on a log scale to emphasize the region close to the body. 


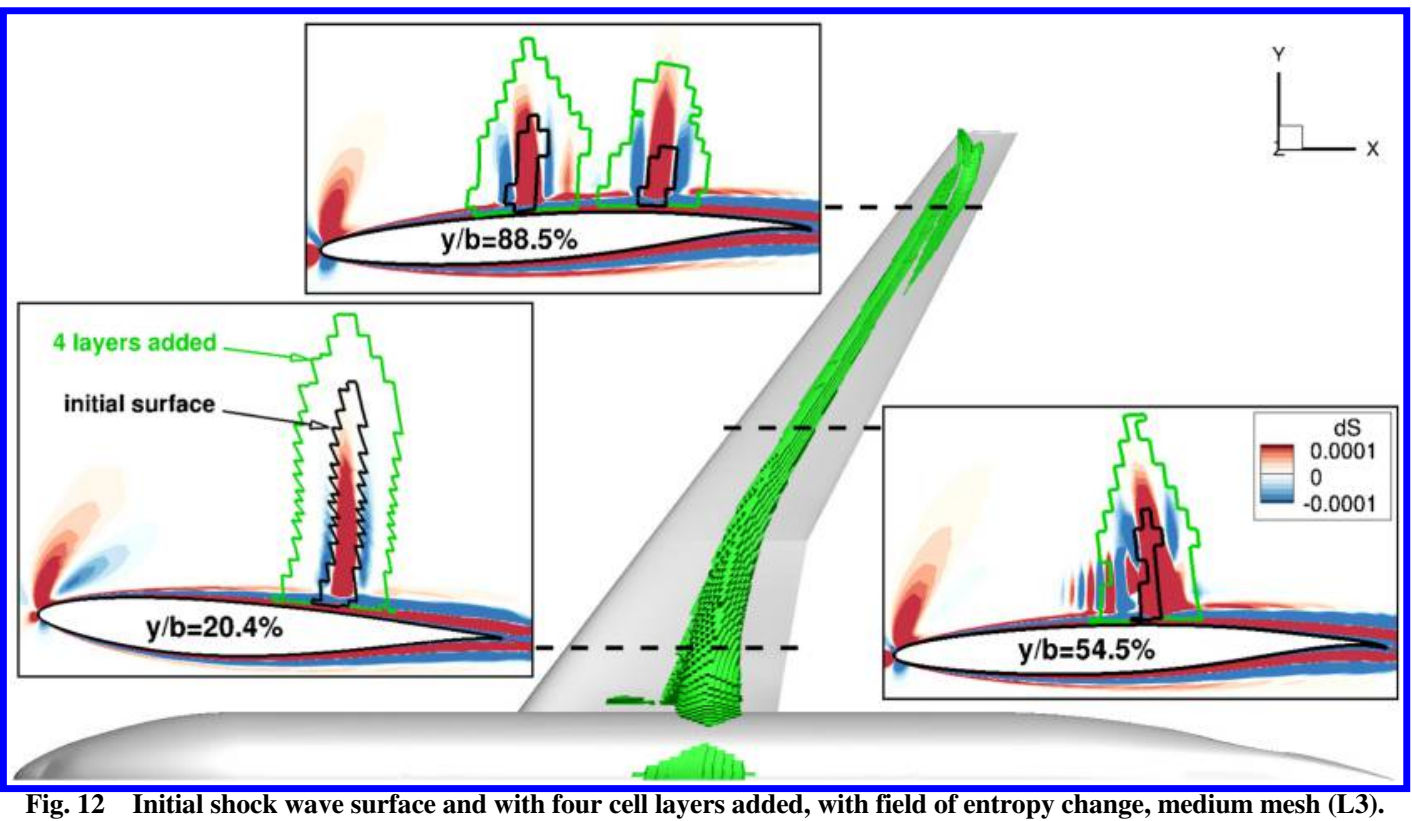

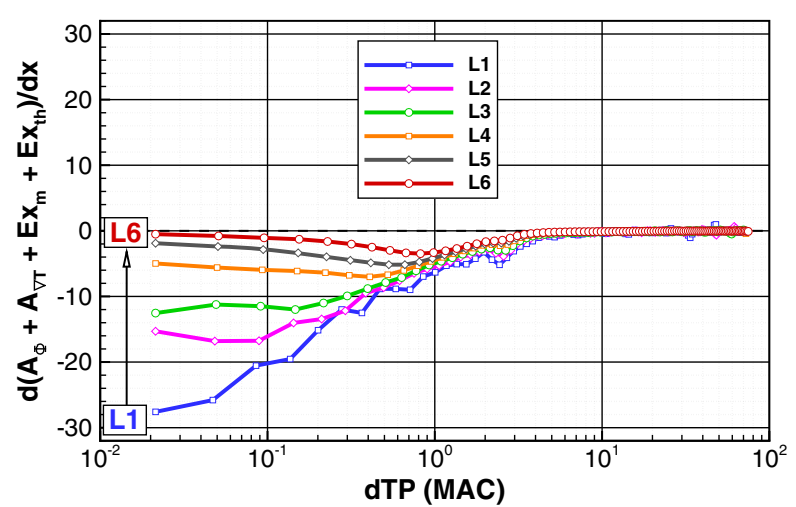

a) Rate of change of the sum of anergy generation and exergy destruction, i.e., Eq. (16a)

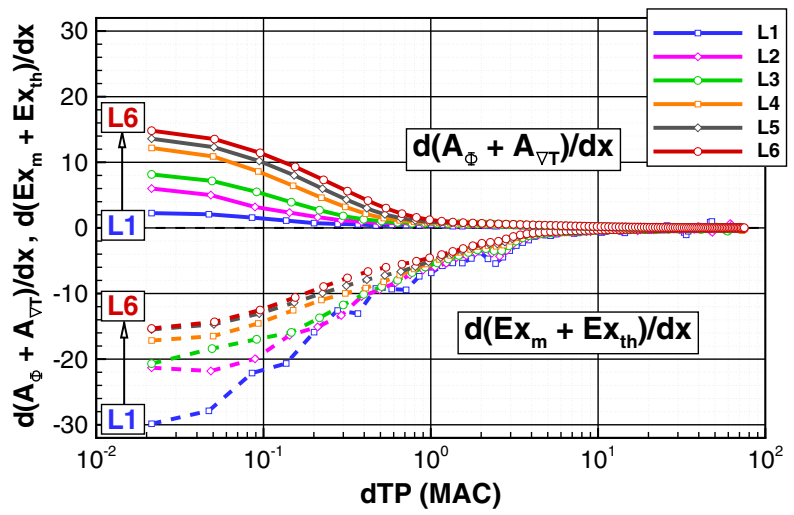

b) Rate of change of viscous + thermal anergy generation and rate of change of exergy destruction, i.e., Eq. (16b)

Fig. 13 Rates of change of anergy generation and exergy destruction vs transverse plane chordwise position, with grid refinement.

Figure $13 \mathrm{a}$ gives the rate of change of the sum of

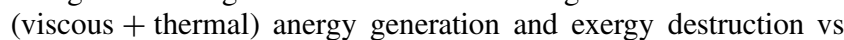
the distance expressed in MACs. The theoretical equality of Eq. (16a) is not numerically verified; the sum is negative up to a few mean aerodynamic chord distances downstream of the body. As the grid is refined, the balance is better verified. Figure $13 \mathrm{~b}$ gives the evolution of the two right-hand-side terms of Eq. (16b), i.e., the rate of anergy generation (solid lines) and the rate of exergy destruction (dashed lines). On the coarsest grid, the rate of exergy destruction is the highest (negative), and the rate of anergy generation is the lowest (positive), leading to the worst balance observed in Fig. 13a. As the grid is refined, the rate of exergy destruction diminishes, and simultaneously the rate of anergy generation increases, thereby providing a better agreement of the overall balance.

To conclude, the exergy destruction downstream of the body is not captured by the terms responsible for anergy production. The viscous and thermal anergy are functions of velocity and temperature gradients squared, respectively. The calculation of these gradients suffers from inaccuracy due to (too coarse) spatial discretization. This study additionally highlights the region where the grid is considered too coarse to numerically satisfy the physical laws; on the finest grid (L6), this region extends up to a few mean aerodynamic chords downstream of the aircraft with a peak error located at $d_{\mathrm{TP}}=0.9 \mathrm{MACs}$. In other words, even for a (rather) clean numerical solution (L6), the incapacity of the code to reconstruct accurate (temperature and velocity) gradients requires a correction for the (thermal and viscous) anergy generation.

\section{Viscous and Thermal Anergy Accuracy Correction}

The underlying idea of the correction is to calibrate the viscous and thermal anergy that would have been generated if the grid was of sufficient quality to compute with a high degree of accuracy the viscous and thermal anergy. To do so, we determine the magnitude of the viscous and thermal anergy that should have been calculated and then attribute to each component the appropriate amount.

a. Error Estimation. The first step consists of determining the amount of missing anergy (not captured). This is achieved by relying on the entropy equation

$$
T_{\infty} \int_{\mathcal{S}_{o}} \rho \delta s(\boldsymbol{V} \cdot \boldsymbol{n}) \mathrm{d} \mathcal{S}=\dot{\mathcal{A}}_{\phi}+\dot{\mathcal{A}}_{\nabla T}+\dot{\mathcal{A}}_{w}+\int_{\mathcal{S}_{O}}-\frac{T_{\infty}}{T}(\boldsymbol{q} \cdot \boldsymbol{n}) \mathrm{d} \mathcal{S}
$$

where the last integral is the anergy associated with the heat transfer across the outer boundary. This theoretical relation is not perfectly numerically satisfied because the viscous anergy $\dot{\mathcal{A}}_{\phi}$ and thermal anergy $\dot{\mathcal{A}}_{\nabla T}$ are underestimated. As a consequence, we can evaluate the anergy (entropy) not captured:

$$
\begin{aligned}
& \Delta \dot{\mathcal{A}}:=T_{\infty} \int_{\mathcal{S}_{O}} \rho \delta s(\boldsymbol{V} \cdot \boldsymbol{n}) \mathrm{d} \mathcal{S} \\
& -\left(\dot{\mathcal{A}}_{\phi}+\dot{\mathcal{A}}_{\nabla T}+\dot{\mathcal{A}}_{w}+\int_{\mathcal{S}_{O}}-\frac{T_{\infty}}{T}(\boldsymbol{q} \cdot \boldsymbol{n}) \mathrm{d} \mathcal{S}\right)
\end{aligned}
$$




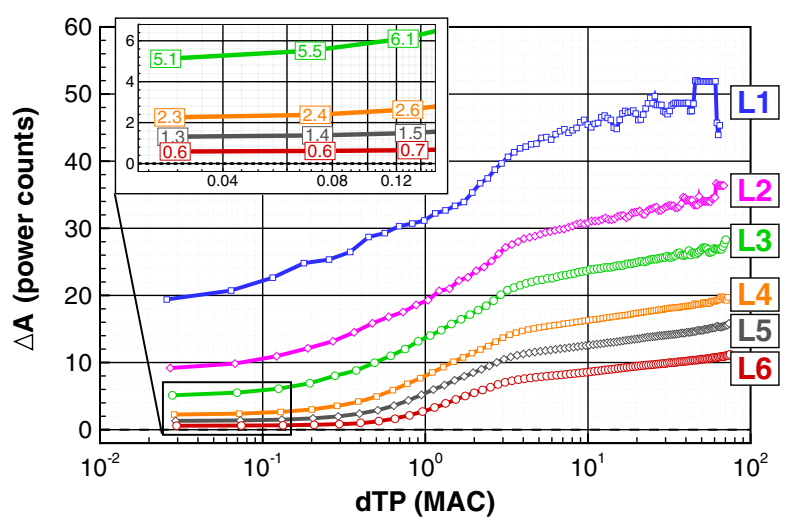

a) Missing anergy $\Delta \dot{\mathcal{A}}$

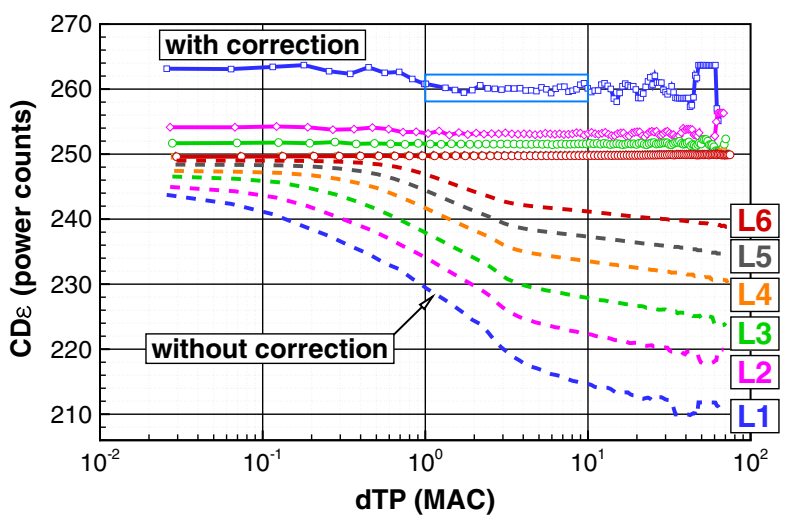

b) Total drag-power coefficient with or without correction

Fig. 14 Determination of the accuracy improvement provided by the correction.

Figure 14a gives the evolution of this quantity in the wake of the airplane as a function of the transverse plane location.

Just downstream of the body, the correction reaches $20 \mathrm{pc}$ for the coarsest mesh (L1) and drastically diminishes with grid refinement: 2.3, 1.3, and $0.6 \mathrm{pc}$ correction on the three finest grids (L4, L5, and L6). The magnitude of the anergy not captured $\Delta \mathcal{A}$ in the (very) near field of the body (for $d_{\mathrm{TP}}<10^{-1} \mathrm{MACs}$ ) is a direct indication of the capacity of the coarse grids to allow for accurate temperature and thermal gradients evaluation in the vicinity of the airplane. For the finest grids, the correction mainly accounts for the dissipation of the lift-induced vortices that is not captured in the far field.

From Fig. 14b, one can see that the correction is very effective to provide a total drag-power coefficient $C D_{\varepsilon}$ independent of the transverse plane position (solid lines). From the coarsest grid, we observe that for $d_{\mathrm{TP}} \leq 1$ mean aerodynamic chord the total drag predicted seems slightly overestimated, while for $10 \leq d_{\mathrm{TP}}$ the coefficient starts oscillating due to spurious entropy variation. In the midfield of the body $1 \leq d_{\mathrm{TP}} \leq 10$, the total drag power coefficient is relatively stable, and therefore this represents the region of confidence in the application of the correction. Without correction (dashed lines), depending on the grid density, this coefficient drops from 14 to $2.5 \mathrm{pc}$ within a distance of only one mean aerodynamic chord.

This correction ensures the consistency of the exergy analysis with the flow solution. But because the total anergy outflow is also subjected to spurious variations, this method does not discriminate the spurious (numerical) anergy from the physical anergy. As a consequence, an accuracy only equivalent to the near-field approach for drag prediction is expected. Note that the correction heavily relies on the accurate definition and calculation of the wave anergy $\dot{\mathcal{A}}_{w}$.

b. Viscous and Thermal Anergy Correction. Now that the total anergy not captured has been identified and effectively improves the total drag power predicted, it must be distributed between the viscous and

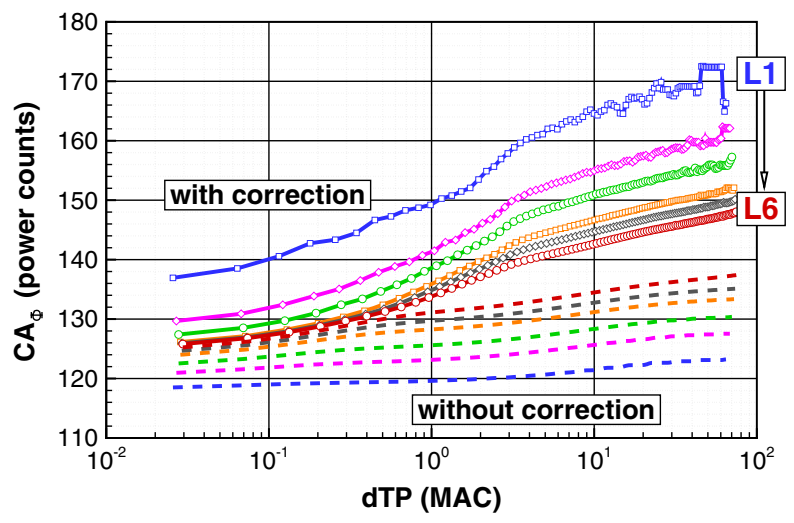

a)Viscous anergy

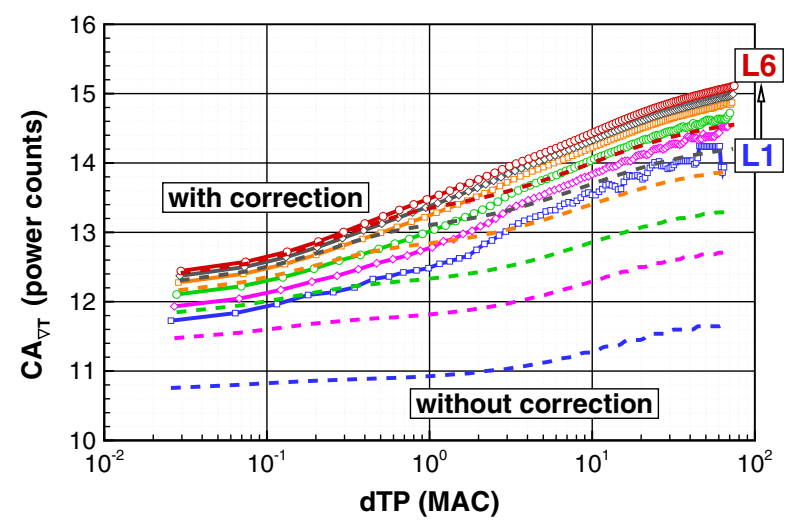

b) Thermal anergy

Fig. 15 Viscous and thermal anergy generation in the wake, with or without correction.

the thermal anergy. The sharing of this quantity between the viscous anergy and the thermal anergy is made as

$$
\begin{gathered}
\dot{\mathcal{A}}_{\Phi}^{c}:=\dot{\mathcal{A}}_{\Phi}+\zeta_{\phi}^{\text {wake }} \times \Delta \dot{\mathcal{A}} \\
\dot{\mathcal{A}}_{\nabla T}^{c}:=\dot{\mathcal{A}}_{\nabla T}+\left[1-\zeta_{\phi}^{\text {wake }}\right] \times \Delta \dot{\mathcal{A}}
\end{gathered}
$$

where $\zeta_{\phi}^{\text {wake }}$ represents the proportion of viscous anergy generated in the wake. The determination of this coefficient is made by evaluating the ratio of viscous anergy to the total anergy generated within the boundary layers $\zeta_{\phi}^{\mathrm{BL}}$. This ratio is higher in the boundary layers than in the wake since the thermal gradients are higher in the boundary layers than in the wake. As a consequence, the following rule of thumb is used for the correction:

$$
\zeta_{\phi}^{\text {wake }}:=\kappa \zeta_{\phi}^{\mathrm{BL}} \quad \text { with } \quad \kappa \geq 1
$$

In the present application, $\kappa$ is taken as a constant but should actually range from 1 at the trailing edge from a wing and rapidly asymptote to provide $\zeta_{\phi}^{\text {wake }} \approx 1$ within a few body lengths. An analysis shows that the dissipation within the boundary layers is due at about $91 \%$ to the viscous dissipation. From the argument that $\zeta_{\phi}^{\text {wake }} \geq \zeta_{\phi}^{\mathrm{BL}}$, a constant value of $\zeta_{\phi}^{\text {wake }}=95 \%$ is chosen, meaning that

$$
\dot{\mathcal{A}}_{\phi}^{c}=\dot{\mathcal{A}}_{\phi}+0.95 \times \Delta \dot{\mathcal{A}}, \quad \dot{\mathcal{A}}_{\nabla T}^{c}=\dot{\mathcal{A}}_{\nabla T}+0.05 \times \Delta \dot{\mathcal{A}}
$$

It could also have been simply assumed that $100 \%$ of the dissipation occurring in the wake contributes to viscous anergy and none contributes to thermal anergy. Figure $\underline{15}$ gives the corresponding 


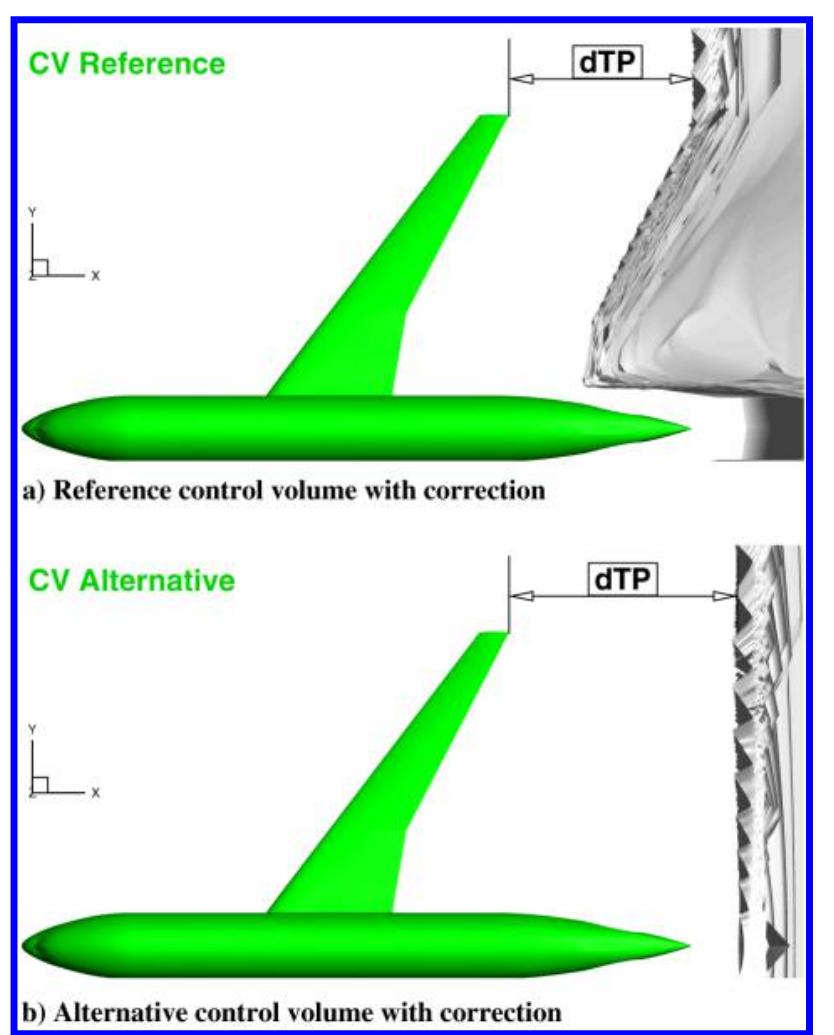

Fig. 16 Control volume definition for drag assessment with correction.

evolution of the viscous (a) and thermal (b) anergy generation with or without correction in the wake.

Without correction (dashed lines), the coefficient is almost flat, the primary value only depending on the grid density. The correction yields coefficients (solid lines) that converge smoothly toward higher values. For the viscous component (a), we observe an overestimation: $\dot{\mathcal{A}}_{\phi}$ is (much) greater on the coarse grid than on the fine grid. As regards the thermal exergy, from the value of $\zeta_{\phi}^{\text {wake }}=95 \%$ chosen, little impact is to be observed.

\section{Drag Prediction and Breakdown}

The aerodynamic assessment of the configuration is discussed and compared with momentum-based methods.

a. Drag Breakdown. The transverse plane is located at a distance of 2.5 MACs; see Fig. 16a. All terms of Eq. (7) are given for all six grid densities in Table $\underline{3}$, expressed in power counts. Viscous and thermal anergy production are presented with or without correction. Also, restated for comparison is the (total) near-field drag coefficient.

As regards exergy outflows, note that the mechanical exergy is much larger than the thermal component. In fact, the magnitude of the mechanical exergy, mainly associated with the transverse kinetic energy of the lift-induced vortices, is directly comparable to the induced drag identified by the far-field drag method of van der Vooren and Destarac; see Table 2. This outcome is, however,

Table 3 Exergy-based drag power breakdown from $f f x$ and mesh refinement, with correction; the near-field drag coefficient serves as reference

\begin{tabular}{lcccccccccc}
\hline \hline & \multicolumn{8}{|c}{ Far-field exergy via ffx } \\
\cline { 2 - 9 } Mesh & $C \dot{\mathcal{E}}_{m}$ & $C \dot{\mathcal{E}}_{\text {th }}$ & $C \dot{\mathcal{A}}_{w}$ & $C \dot{\mathcal{A}}_{\phi}$ & $C \dot{\mathcal{A}}_{\phi}^{c}$ & $C \dot{\mathcal{A}}_{\nabla T}$ & $C \dot{\mathcal{A}}_{\nabla T}^{c}$ & $C D_{\varepsilon}$ & $C D_{\varepsilon}^{c}$ & $C D_{\mathrm{nf}}$ \\
\hline L1 & 85.0 & 3.4 & 3.3 & 120.1 & 155.3 & 11.0 & 12.9 & 222.8 & 259.9 & 259.7 \\
L2 & 86.1 & 3.4 & 3.0 & 122.2 & 147.4 & 11.7 & 13.2 & 226.4 & 253.1 & 253.0 \\
L3 & 87.3 & 3.4 & 3.3 & 126.4 & 144.2 & 12.5 & 13.4 & 232.9 & 251.6 & 251.4 \\
L4 & 88.2 & 3.4 & 3.5 & 129.1 & 141.0 & 13.0 & 13.6 & 237.2 & 249.7 & 249.8 \\
L5 & 89.3 & 3.5 & 3.7 & 130.6 & 139.6 & 13.3 & 13.7 & 240.4 & 249.8 & 249.9 \\
L6 & 90.4 & 3.6 & 3.8 & 132.2 & 138.2 & 13.5 & 13.9 & 243.5 & 249.9 & 249.8 \\
\hline \hline
\end{tabular}

fortunate as being dependent on the transverse plane location. One must also recognize that the lift has a global impact on the flow [50], meaning that a clear and unambiguous definition of vortex drag is not straightforward.

As regards anergy production, first note that the wave anergy is in excellent agreement with the wave drag predicted by this well-tried method. The viscous dissipation is one order of magnitude greater than the thermal dissipation, as expected from the qualitative assessment of Fig. 9. The drag predicted without correction for this control volume far from the body is largely underestimated, as highlighted in Fig. 14b. The correction ranges from $37.1 \mathrm{pc}$ on the coarsest grid to $6.4 \mathrm{pc}$ on the finest grid, which are significant values. With correction, the total drag (power) predicted is in excellent agreement with the near-field drag method; see the last column.

In addition, to this very satisfactory improvement, the correction relaxes the constraint on the control volume definition which, for such complex geometries, can become a challenging and timeconsuming task. In fact, a very similar accuracy would be obtained with the control volume depicted in Fig. 16b. As a consequence, the postprocessing with the code $f f x$ of any complex geometry becomes more robust and effective.

To conclude this aerodynamic assessment from an exergy/anergy standpoint, mechanical aspects (exergy and anergy) represent about $91 \%$ of the total anergy (or drag power) that the airplane involves. Then, thermal aspects (exergy and anergy) are second-order contributors to the drag, around $7 \%$. Mach number sensitivity analysis shows that they are negligible at low speed and never exceed $10 \%$ of the total drag [19]. Finally, the wave anergy accounts for the remaining $2 \%$. This approach also highlights that only $62 \%$ of the total anergy has been produced at a distance of 2.5 MACs downstream of the airplane. In other words, there is a large recoverable work potential present in the wake of the airplane.

b. Comparison to Momentum-Based Methods. The results from the drag assessment with or without correction are graphically represented in Fig. 17 with reference to the fifth DPW CFD median value of $249.6 \mathrm{dc}$ and a standard deviation of $5.3 \mathrm{dc}$ [21]. As mentioned in the Introduction, experimental wind-tunnel testing campaigns were made in two different facilities, yielding a total drag coefficient of $248 \mathrm{dc}$ (NTF [39]) and $241 \mathrm{dc}$ (Ames Research Center [40]). The difference between the NTF and Ames data is about seven counts, which is similar to the standard deviation of the CFD data. Comparison of the results to wind-tunnel data is reasonable, within about four counts to the median solution. This is only considered as an indication because the wind-tunnel test and CFD problem setups are inherently different, meaning that there is some question as to how well they should agree [21]. Also shown for reference are the total drags predicted from the two momentum-based methods implemented in the code $f f d 72$.

The best obtainable results without correction $\left(C D_{\varepsilon}\right.$, dashed line) are within the standard deviation but fail to converge even on the finest grid. \pm As a consequence of this inability, a numerical correction was introduced. With it, the distance relative to the airplane becomes less crucial, and the transverse plane can be located, for example, at a distance of 2.5 MACs $\left(C D_{\varepsilon}^{c}\right.$, solid line). With correction, the total drag predicted is found to be as sensitive to grid density as the near-field drag method $\left(C D_{\text {nf }}\right)$. From this latter result, we conclude that the postprocessing code is capable of providing a flow analysis that is consistent with the accuracy of the flow solution; this critical point validates the numerical implementation of the exergybased formulation. All methods converge precisely toward the DPW CFD median value and are well within the standard deviation, the far-field drag method $\left(C D_{\mathrm{ff}}\right)$ being the most efficient approach. A qualitative good agreement with wind-tunnel experimental data reinforces this overall satisfactory drag prediction.

\footnotetext{
${ }^{\dagger}$ If the correction is not activated, the transverse plane should be located as close as possible to the body to avoid numerical dissipation. Even for the finest mesh and for the shortest distance, the total drag power predicted is $0.6 \mathrm{pc}$ too low; see first data point of Fig. 14a.
} 


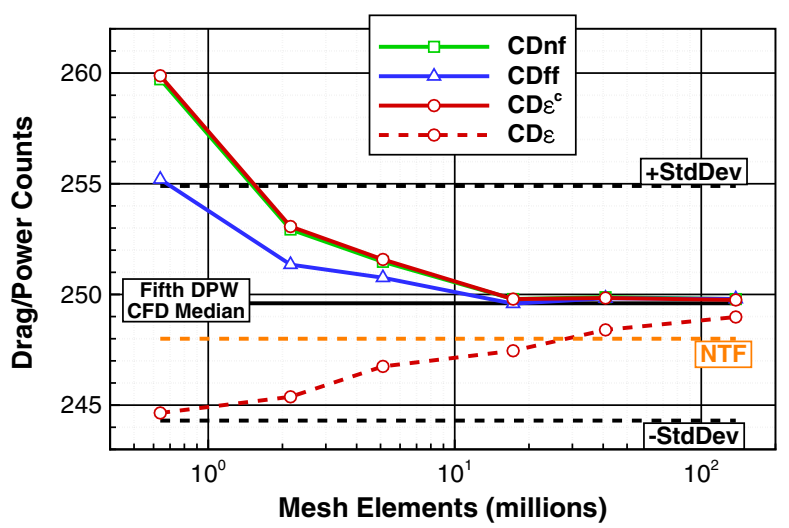

Fig. 17 Exergy-based drag-power breakdown from $f f x$ with mesh refinement.

It has been shown that on the coarse grids the missing anergy $\Delta \dot{\mathcal{A}}$ accounts for excessive exergy destruction (or equivalently spurious entropy generation) and thereby leads to an overestimation of the total drag experienced by the body equivalent to the near-field drag method. Consequently, an enhancement of the correction could be to subtract from the missing anergy the spurious drag $C D_{\mathrm{sp}}$ identified by the far-field drag method. By doing so, an accuracy similar to the farfield drag method of Destarac [24] and van der Vooren and Destarac [25] would be obtained. However, because this spurious drag is identified thanks to a very different approach, this additional correction is not adopted here.

\section{Conclusions}

To validate the accuracy and robustness of the ONERA postprocessing code $f f x$ in which a new exergy method has been implemented, an industry-relevant test case was investigated. It corresponds to the NASA Common Research Model that is representative of a Boeing B777-200. The airplane was studied in cruise conditions: transonic regime at cruise $\mathrm{CL}=0.5$. These settings correspond to case 1 of the Fifth Drag Prediction Workshop, the committee of which provided a family of six multiblock and structured grids for the drag convergence study. As the workshop brought together more than 50 participants, a solid numerical database for validation is available.

As regards drag assessment, a reference control volume was defined to be close to the body. An analysis showed that the theoretical balance between exergy destruction and anergy generation was not numerically satisfied well in the wake, this observation being worsened with grid coarsening. The main reason identified is an underestimation of the temperature and velocity gradients. As a consequence, a correction of the numerical accuracy of the viscous and thermal anergy was adopted. It was calibrated by considering that, for a transonic regime, $95 \%$ of the dissipation in the wake would contribute to viscous anergy and only $5 \%$ would contribute to thermal anergy. Also, the shock wave treatment yielded coefficients in good agreement with the far-field drag method. The procedure ensured results largely independent of the surface encompassing the shock system(s), which solves the difficult separation of viscous and wave drag encountered by many far-field drag methods [35].

With correction of the viscous and thermal anergy generation, an accuracy similar to the near-field drag method was achieved, and the robustness of the code was improved. Also in reasonable agreement with experimental wind-tunnel testing data $[39,40]$, these satisfactory results enabled the validation of the exergy-based formulation and its numerical implementation in the code $f f x$ for the drag prediction of transonic flows over complex three-dimensional geometries. An accuracy equivalent to the far-field drag method of van der Vooren and Destarac could be obtained by removal of the spurious drag identified by this latter method.

Perspectives include parallel processing to reduce computational cost and improvement in the calculation of the temperature and velocity gradients to enhance the precision of the code.

\section{Acknowledgments}

This work is supported by the French National Research Agency. The author is indebted to O. Atinault and A. Merlen for supervising the $\mathrm{Ph} . \mathrm{D}$. in which the present methodology and postprocessing code were developed. D. Destarac is acknowledged for his advice regarding the far-field drag prediction methods and for his help regarding the numerical implementation of the exergy-based formulation.

\section{References}

[1] Gohardani, A. S., Doulgeris, G., and Singh, R., "Challenges of Future Aircraft Propulsion: A Review of Distributed Propulsion Technology and Its Potential Application for the All Electric Commercial Aircraft," Progress in Aerospace Sciences, Vol. 47, No. 5, 2011, pp. 369-391. doi:10.1016/j.paerosci.2010.09.001

[2] Ashcraft, S. W., Padron, A. S., Pascioni, K. A., Stout, G. W., Jr., and Huff, D. L., "Review of Propulsion Technologies for $N+3$ Subsonic Vehicle Concepts," NASA TM-2011-217239, 2011.

[3] Covert, E. E., James, C. R., Kimzey, W. F., Richey, G. K., and Rooney, E. C., Thrust and Drag: Its Prediction and Verification, edited by Covert, E. E., Progress in Astronautics and Aeronautics, AIAA, New York, 1985.

[4] Stoliker, F. N., "Guide to In-Flight Thrust Measurement of Turbojets and Fan Engines," Advisory Group for Aerospace Research and Development, Ministry-Industry Drag Analysis Panel Study Group, AG-237, 1979.

[5] Kawai, R. T., Friedman, D., and Serrano, L., "Blended Wing Body (BWB) Boundary Layer Ingestion (BLI) Inlet Configuration and System Studies," NASA CR-2006-214534, 2006.

[6] Liu, C., Doulgeris, G., Laskaridis, P., and Singh, R., "Thermal Cycle Analysis of Turboelectric Distributed Propulsion System with Boundary Layer Ingestion,” Aerospace Science and Technology, Vol. 27, No. 1, 2013, pp. 163-170. doi:10.1016/j.ast.2012.08.003

[7] Felder, J., Kim, H. D., and Brown, G., "Turboelectric Distributed Propulsion Engine Cycle Analysis for Hybrid-Wing-Body Aircraft," 47th AIAA Aerospace Sciences Meeting Including the New Horizons Forum and Aerospace Exposition, AIAA Paper 2009-1132, Jan. 2009.

[8] Arntz, A., Atinault, O., and Merlen, A., "Exergy-Based Formulation for Aircraft Aeropropulsive Performance Assessment: Theoretical Development," AIAA Journal, Vol. 53, No. 6, 2015, pp. 1627-1639. doi:10.2514/1.J053467

[9] Rant, Z., "Exergie, ein Neues Wort für Technische Arbeitsfähigkeit," Forschung im Ingenieurwesen, Vol. 22, No. 1, 1956, pp. 36-37.

[10] Moran, M. J., and Sciubba, E., "Exergy Analysis: Principles and Practice," Journal of Engineering for Gas Turbines and Power, Vol. 116, No. 2, 1994, pp. 285-290. doi: $10.1115 / 1.2906818$

[11] Roth, B., and Mavris, D., "Minimizing Vehicle Environmental and Economic Cost via Thermodynamic Work Potential," National Science Foundation DMII Grantees Conference, 2001.

[12] Alabi, K., Ladeinde, F., von Spakovsky, M., Moorhouse, D., and Camberos, J. A., "The Use of the 2nd Law as a Potential Design Tool for Aircraft Air Frame Subsystems," International Journal of Thermodynamics, Vol. 9, No. 4, 2006, pp. 1-14.

[13] Alabi, K., and Ladeinde, F., "Utilizing CFD-Based Exergy Calculations in the Design/Optimization of a Complete Aircraft System," 45th AIAA Aerospace Sciences Meeting and Exhibit, AIAA Paper 2007-1130, Jan. 2007.

[14] Li, H., and Figliola, R., "Exergy Based Design Methodology for Airfoil Shape Optimization," 10th AIAA/International Society for Structural and Multidisciplinary Optimisation Multidisciplinary Analysis and Optimization Conference, AIAA Paper 2004-4632, 2004.

[15] Li, H., Stewart, J., and Figliola, R., "Exergy Based Design Methodology for Airfoil Shape Optimization and Wing Analysis," 25th International Congress of the Aeronautical Sciences, 2006.

[16] Monsch, S., Li, H., Harris, R., Stewart, J., Figliola, R., and Camberos, J., "Exergy Based Design Methodology for Wing Shape Optimization and Analysis," 39th AIAA Thermophysics Conference, AIAA Paper 20074053, June 2007.

doi: $10.2514 / 6.2007-4053$

[17] Arntz, A., Atinault, O., and Destarac, D., "Numerical Airframe Aerodynamic Performance Prediction: An Exergy Point of View," 49th International Symposium of Applied Aerodynamics, Assoc. Aeronautique et Astronautique de France, FP04-2014-arntz, March 2014. 
[18] Arntz, A., Atinault, O., Destarac, D., and Merlen, A., "Exergy-Based Aircraft Aeropropulsive Performance: CFD Application to Boundary Layer Ingestion," 32nd AIAA Applied Aerodynamics Conference, AIAA Paper 2014-2573, June 2014.

[19] Arntz, A., "Civil Aircraft Aero-Thermo-Propulsive Performance Assessment by an Exergy Analysis of High-fidelity CFD-RANS Flow Solutions," Ph.D. Dissertation, Univ. de Lille 1-Sciences et Technologies, Lille, France, Nov. 2014.

[20] Arntz, A., and Atinault, O., "Exergy-Based Performance Assessment of a Blended Wing-Body with Boundary-Layer Ingestion," AIAA Journal (to be published). doi:10.2514/1.J054072

[21] Levy, D. W., Laflin, K. R., Tinoco, E. N., Vassberg, J. C., Mani, M., Rider, B., Rumsey, C. L., Wahls, R. A., Morrison, J. H., Brodersen, O. P., Crippa, S., Mavriplis, D. J., and Murayama, M., "Summary of Data from the Fifth Computational Fluid Dynamics Drag Prediction Workshop," AIAA Journal, Vol. 51, No. 4, 2014, pp. 1194-1213. doi:10.2514/1.C032389

[22] Van Dam, C. P., "Recent Experience with Different Methods of Drag Prediction," Progress in Aerospace Sciences, Vol. 35, No. 8, 1999, pp. 751-798. doi:10.1016/S0376-0421(99)00009-3

[23] Betz, A., "Ein Verfahren zur Direkten Ermittlung des Profilwiderstandes," Zeitschrift für Flugtechnik un Motorluftschiffahrt, Vol. 16, 1925, pp. 42-44.

[24] Destarac, D., "Far-Field/Near-Field Drag Balance and Applications of Drag Extraction in CFD," CFD-Based Aircraft Drag Prediction and Reduction Lecture Series, Von Kármán Inst. for Fluid Dynamics, Hampton, VA, 2003, pp. 1-65.

[25] Van der Vooren, J., and Destarac, D., "Drag/Thrust Analysis of JetPropelled Transonic Transport Aircraft; Definition of Physical Drag Components," Aerospace Science and Technology, Vol. 8, No. 7, 2004, pp. 556-671. doi:10.1016/j.ast.2004.09.001

[26] Camberos, J. A., and Doty, J. H., "Fundamentals of Exergy Analysis," Exergy Analysis and Design Optimization for Aerospace Vehicles and Systems, edited by Moorhouse, D. J., and Camberos, J. A., Progress in Astronautics and Aeronautics, AIAA, Reston, VA, 2011, pp. 9-76, Chap. 2.

[27] Cengel, Y. A., and Boles, M. A., Thermodynamics: An Engineering Approach, 5th ed., McGraw-Hill Higher Education, Boston, 2006, pp. 423-485, Chap. 8.

[28] De Oliveira, S., Jr., Exergy: Production, Cost and Renewability, 1st ed., Springer-Verlag, London, 2013, pp. 5-53, 237-280, Chaps. 2, 8

[29] Roth, B., "Aerodynamic Drag Loss Chargeability and Its Implications in the Vehicle Design Process," 1st AIAA Aircraft, Technology, Integration, and Operations Forum, AIAA Paper 2001-5236, 2001.

[30] Oswatitsch, K., Gas Dynamics, Academic Press, New York, 1956, Chaps. 4, 11.

[31] Drela, M., "Power Balance in Aerodynamics Flows," AIAA Journal, Vol. 47, No. 7, 2009, pp. 1761-1771. doi: $10.2514 / 1.42409$

[32] Giles, M. B., and Cummings, R. M., "Wake Integration for ThreeDimensional Flowfield Computations: Theoretical Development," Journal of Aircraft, Vol. 36, No. 2, 1999, pp. 357-365. doi: $10.2514 / 2.2465$

[33] Van der Vooren, J., and Slooff, J. W., "CFD-Based Drag Prediction: State-of-the-Art, Theory, and Prospects," Lectures Notes, AIAA Professional Studies Series, Course on Drag-Prediction and Measurement, Dutch Aerospace Lab, Nationaal Lucht- en Ruimtevaartlaboratorium, TP-90247, Amsterdam, 1990.

[34] Paparone, L., and Tognaccini, R., "Computational Fluid DynamicsBased Drag Prediction and Decomposition," AIAA Journal, Vol. 41,
No. 9,2003 , pp. $1647-1657$.

doi: $10.2514 / 2.7300$

[35] Ueno, M., Yamamoto, K., Tanaka, K., Murayama, M., and Tognaccini, R., "Far-Field Drag Analysis of NASA Common Research Model Simulation," Journal of Aircraft, Vol. 50, No. 2, 2013, pp. 388-397. doi:10.2514/1.C031766

[36] Chao, D. D., and van Dam, C. P., "Wing Drag Prediction and Decomposition," Journal of Aircraft, Vol. 43, No. 1, 2006, pp. 82-90. doi:10.2514/1.12311

[37] Moore, J., and Moore, J. G., "Entropy Production Rates from Viscous Flow Calculations, Part I. A Turbulent Boundary Layer Flow," American Society of Mechanical Engineers Gas Turbine Conference, American Soc. of Mechanical Engineers Paper 1983-GT-70, Phoenix, AZ, 1983.

[38] Vassberg, J. C., DeHaan, M. A., Rivers, S. M., and Wahls, R. A., "Development of a Common Research Model for Applied CFD Validation Studies," 26th AIAA Applied Aerodynamics Conference, AIAA Paper 2008-6919, 2008.

[39] Rivers, M. B., and Dittberner, A., "Experimental Investigation of the NASA Common Research Model," 28th AIAA Applied Aerodynamics Conference, AIAA Paper 2010-4218, 2010.

[40] Rivers, M. B., and Dittberner, A., "Experimental Investigation of the NASA Common Research Model in the NASA Langley National Transonic Facility and NASA Ames 11-Ft Transonic Wind Tunnel," 49th AIAA Aerospace Sciences Meeting, AIAA Paper 2011-1126, 2011.

[41] Cambier, L., Heib, S., and Plot, S., "The Onera elsA CFD Software: Input from Research and Feedback from Industry," Mechanics and Industry, Vol. 14, No. 3, 2013, pp. 159-174. doi:10.1051/meca/2013056

[42] Jameson, A., Schmidt, W., and Turkel, E., "Numerical Solution of the Euler Equations by Finite Volume Methods Using Runge Kutta Time Stepping Schemes," 14th Fluid and Plasma Dynamics Conference, AIAA Paper 1981-1259, 1981.

[43] Spalart, P. R., and Allmaras, S. R., "A One-Equation Turbulence Model for Aerodynamic Flows," Recherche Aerospatiale, No. 1, 1994, pp. 5-21.

[44] Hue, D., "Fifth Drag Prediction Workshop: Computational Fluid Dynamics Studies Carried Out at ONERA," AIAA Journal, Vol. 51, No. 4, 2014, pp. 1295-1310. doi:10.2514/1.C032054

[45] Patterson, J. C., Jr., and Flechner, S. G., "Exploratory Wind-Tunnel Investigation of a Wingtip-Mounted Vortex Turbine for Vortex Energy Recovery," NASA Technical Paper 2468, 1985.

[46] Abeyounis, W. K., Patterson, J. C., Jr., Stough, H. P., III, Wunschel, A. J., and Curran, P. D., "Wingtip Vortex Turbine Investigation for Vortex Energy Recovery," Society of Automotive Engineers Aerotech '90, 1990 Society of Automotive Engineers Aerospace Technology Conference and Exposition, Soc. of Automotive Engineers, Oct. 1990.

[47] Vachon, M. J., Ray, R. J., Walsh, K. R., and Ennix, K., "F/A-18 Performance Benefits Measured During the Autonomous Formation Flight Project," NASA TM-2003-210734, 2003.

[48] Gariépy, M., Malouin, B., Trépanier, J.-Y., and Laurendeay, E., "FarField Drag Decomposition Applied to the Drag Prediction Workshop 5 Cases," Journal of Aircraft, Vol. 50, No. 6, 2013, pp. 1822-1831. doi:10.2514/1.C032204

[49] Lovely, D., and Haimes, R., "Shock Detection from Computational Fluid Dynamics Results," AIAA Paper 1999-33493, 1999.

[50] McLean, D., "Wingtip Devices: What They Do and How They Do It?" Boeing Performance and Flight Operations Engineering Conference, 2005.

Z. Rusak

Associate editor 